\title{
Efficacy of Three Antimalarial Regimens for Uncomplicated Plasmodium Falciparum Malaria in Cambodia, 2009 - 2011: A Randomized Control Trial
}

Dysoley Lek ( $\nabla$ soleycnm@gmail.com )

National Center for Parasitology Entomology and Malaria control, Cambodia

Agus Rachmat

US Naval Medical Research Unit No 2

Dustin Harrison

US Naval Medical Research Unit No 2

Geoffrey Chin

Uniformed Services University: Uniformed Services University of the Health Sciences

Suwanna Chaoratanakawee

Mahidol University Faculty of Public Health

David Saunders

Uniformed Services University: Uniformed Services University of the Health Sciences

Didier Menard

Institut Pasteur du Cambodge

William O. Rogers

US Naval Medical Research Unit No 2

Research Article

Keywords: Uncomplicated Falciparum Malaria, Cambodia, Antimalarial Resistance, Randomized Clinical Trial, Therapeutic Efficacy

Posted Date: December 28th, 2021

DOI: https://doi.org/10.21203/rs.3.rs-1177741/v1

License: (c) (7) This work is licensed under a Creative Commons Attribution 4.0 International License. Read Full License 


\section{Abstract}

Background: Antimalarial resistance remains an important public health challenge in Cambodia. The effectiveness of three therapies for uncomplicated Falciparum malaria were evaluated in Oddar Meanchey province in Northern Cambodia from 2009 - 2011.

Methods: In this randomized, open-label, parallel group controlled trial, 211 subjects at least 5 years old with uncomplicated Falciparum malaria were treated with directly observed therapy. Over 3 days, 63 received artesunate-mefloquine (AS/MQ), 77 received dihydroartemisinin-piperaquine (DHA/PPQ), and 71 received atovaquone-proguanil (ATQ/PG). Subjects were followed for 42 days or until recurrent parasitemia. Genotyping of $m s p 1, m s p 2$, and glurp among individual parasite isolates distinguished recrudescence from reinfection. Pfmdr1 copy number was measured by real-time PCR and half-maximal parasite inhibitory concentrations $\left(\mathrm{IC}_{50}\right)$ was measured in vitro by 48-hour isotopic hypoxanthine incorporation assay.

Results: The primary outcome of per-protocol PCR-adjusted efficacy at 42 days was analyzed for 190 (90.0\%) of the enrolled subjects. PCR-adjusted efficacy (95\% confidence interval) at 42 days was $80.6 \%$ (70.8 - 90.5\%) for AS/MQ, 97.2\% (93.3 - 100\%) for DHA/PPQ, and 92.9\% (86.1 - 99.6\%) for ATQ/PG. On day $3,59.3 \%$ remained parasitemic. At baseline, $46.9 \%$ had microscopic $P$. falciparum gametocytemia. Both recurrences in the DHA/PPQ arm lost $P$ fmdr1 copy number amplification at recrudescence. All four recurrences in the ATQ/PG arm were wild-type for cytochrome bc ${ }_{1}$. One subject withdrew from the ATQ/PG arm due to drug allergy.

Conclusions: This previously unpublished study was conducted at the epicenter of substantial multi-drug resistance that emerged soon thereafter. Occurring early in the national transition from AS/MQ to DHA/PPQ, both DHA/PPQ and ATQ/PG had acceptable efficacy against uncomplicated falciparum malaria. However, efficacy of AS/MQ was only $80 \%$ with apparent mefloquine resistance based on elevated Pfmdr 1 copy number and IC F0. $_{5 y} 2009$, there was already significant evidence of artemisinin resistance not previously reported at the Northern Cambodia-Thai border. This study suggests the basis for early development of significant DHA/PPQ failures within 3 years of introduction. Artemisinin resistance likely occurred on the Northern border concurrently with that reported along the Western border in Pailin.

Trial Registration: This legacy trial was conducted prior to International Committee of Medical Journal Editors' requirements for preregistration on ClinicalTrials.gov. The full protocol has been provided.

\section{Background}

The spread of drug resistant $P$. falciparum has complicated efforts to control malaria. This may lead to unnecessary mortality if ineffective drugs remain the standard of care after drug-resistant strains become established $(1,2)$. In Southeast Asia, resistance is common to multiple antimalarial drugs including chloroquine (CQ), sulfadoxine-pyrimethamine, quinine (QN) and mefloquine (MQ) (3). Given the worsening situation, countries in the region adopted shortcourse artemisinin combination therapies (ACT) as first-line treatment for uncomplicated $P$. falciparum malaria. In Cambodia, current Ministry of Health guidelines have returned to the use of artesunate-mefloquine (AS/MQ) as first-line therapy for uncomplicated $P$. falciparum malaria. This followed several years where dihydroartemisin-piperaquine (DHA/PPQ) had replaced AS/MQ due to widespread clinical resistance to the latter, and evidence of inverse resistance patterns to the two ACTs (4). Despite the recent return to AS/MQ there is already growing evidence of resistance to AS/MQ at several sites in Cambodia and neighboring Thai provinces.

Mefloquine was first introduced along the Thai-Cambodian border in 1983 (5). In 1994, AS/MQ became the first ACT used along the Thai-Burmese border due to increasing MQ resistance (6). By 1995, AS/MQ became first-line therapy for uncomplicated Falciparum malaria in Thailand, with Cambodia following in 2000 (5). Due to rising AS/MQ failure rates, Western Cambodia adopted DHA/PPQ in 2008, with the rest of the country following in 2012 (7). Shortly after significant DHA/PPQ failures were reported in Oddar Meanchey province (8), Cambodia transitioned back to AS/MQ in regions of significant DHA/PPQ failures with low Pfmdr1 copy numbers (9).

Significant clinical AS/MQ failures were documented in 2003 (10). A regimen of twice daily divided doses of mefloquine (25 mg/kg) and oral artesunate (12 $\mathrm{mg} / \mathrm{kg}$ ) produced 28-day adequate clinical and parasitological response (ACPR) in only $78.6 \%$ of patients with uncomplicated Falciparum malaria in Trat, Thailand. Three other sites in Thailand along the Cambodian border using the same regimen had $>90 \%$ efficacy at the time (10). In 2002 in Pailin, a Western Cambodian province bordering Trat province in Thailand, a similar regimen produced 28-day ACPR in $85.7 \%$ of subjects (11). In that study, nearly one-third of subjects received less than $12 \mathrm{mg} / \mathrm{kg}$ artesunate and $20 \mathrm{mg} / \mathrm{kg}$ mefloquine, because blister pack doses were administered according to age rather than weight. Using the same dosing scheme at sites outside of Pailin, ACPR remained $>90 \%$. Unfortunately by 2004 , despite using weight-based dosing, the 42 -day ACPR dropped to 79.3\% in Pailin (11). In 2006 - 2007 in Chumkiri, a site in southern Cambodia, far from the Thai border, the Naval Medical Research Unit 2 (NAMRU-2) found that a regimen of $12 \mathrm{mg} / \mathrm{kg}$ artesunate over three days and $25 \mathrm{mg} / \mathrm{kg}$ mefloquine on day 0 resulted in 42 -day ACPR in only $81.2 \%$ of subjects (12). Both the Thai and Cambodian studies used directly observed therapy, and the Cambodian studies also used PCR correction for re-infection (13). These findings indicated that resistance to AS/MQ was emerging at the Thai-Cambodian border and may have begun spreading beyond the immediate border area.

The Cambodian Ministry of Health took significant steps to contain the possible spread of $P$. falciparum resistant to AS/MQ. In collaboration with the World Health Organization (WHO) and the Gates Foundation, the Ministry of Health designed a program to confirm the presence of artemisinin resistance, and to contain its spread. The plan defined Phase 1 and Phase 2 containment and elimination zones (Figure 1), including areas where significant failure of AS/MQ was documented (Phase 1). Phase 2 areas adjacent to Phase 1 were thought to be at higher risk. In the Phase 1 containment area, AS/MQ was replaced by dihydroartemisinin-piperaquine (DHA/PPQ) as first-line therapy for uncomplicated $P$. falciparum malaria. The efficacy and tolerability of DHA/PPQ was previously reviewed in 2007 (4) with 14 studies involving 2,636 patients with uncomplicated $P$. falciparum malaria having 28 day cure rates of $97-98 \%$. Therapy was well tolerated with common adverse events of nausea, vomiting, anorexia, headache, dizziness, diarrhea, and abdominal pain occurring in 1 - 
$10 \%$ of subjects - notably also common symptoms of malaria. There were no serious adverse events reported. At the time of the study, the Ministry of Health conducted a large-scale screening and treatment campaign in the Phase 1 zones using PCR for diagnosis of inapparent infections and atovaquone-proguanil (ATQ/PG) therapy.

The success of the containment program was dependent on the extent to which AS/MQ had already spread beyond the Phase 1 containment zone, and on the continuing efficacy of DHA/PPQ and ATQ/PG in treating uncomplicated $P$. falciparum in the Phase 2 containment zone. In previous studies, NAMRU-2 identified sites at risk for AS/MQ resistance by screening approximately 700 parasite samples from cases of uncomplicated Falciparum malaria from 5 provinces within and outside the containment zones for the prevalence of amplified Pfmdr1 genes. Amplifications at this locus are strongly associated with in vivo resistance to mefloquine (14). The highest average Pfmdr1 copy number ( 2.5) was found, unsurprisingly, at Chumkiri, the site in southern Cambodia where NAMRU-2 previously identified significant resistance to AS/MQ (12). Sites in eastern Cambodia, outside both containment zones, had average Pfmdr1 copy numbers $<1.5$, but a site in containment zone 2, Trapeang Prasat, Oddar Meanchey province, had an elevated mean Pfmdr1 copy number ( 2), suggesting a risk of AS/MQ resistance (unpublished data). This study was undertaken in 2009 to assess the in vivo efficacy of $A S / M Q, D H A / P P Q$, and ATQ/PG at this site, following guidelines recommended by WHO and the World Wide Antimalarial Resistance Network (WWARN) $(13,15)$.

\section{Methods Design}

This study was an open-label, parallel group, randomized control trial evaluating the efficacy of 3 standard antimalarial regimens for the treatment of uncomplicated falciparum malaria. Subjects who agreed to participate in the study were randomized to one of three arms - AS/MQ $12 / 25 \mathrm{mg} / \mathrm{kg}$ (max $600 / 1250 \mathrm{mg}$ ), DHA/PPQ 120/960 mg or ATQ/PG 1000/400 mg. Subjects were treated with directly observed therapy for 3 days and followed for up to 42 days or until evidence of recurrent parasitemia. The protocol has been provided as Additional File 1.

\section{Study Location}

The study was conducted at the Trapeang Prasat Health Center in Oddar Meanchey Province, Cambodia (Figure 1). This site is in a rural, agricultural area about 8 hours by road from Phnom Penh. Oddar Meanchey province was considered to be in the Phase 2 containment zone and falciparum malaria was common in the area around the health center. The health center participated in a surveillance program to measure the prevalence of $P$. falciparum molecular drug resistance markers.

\section{Study Enrollment}

Subjects were recruited from areas around the study site and included if they fulfilled the following criteria: 1 ) age $\geq 5$ years, 2) blood-stage $P$. falciparum parasitemia $>1000$ but $<100,000$ parasites per $\mu \mathrm{L}, 3$ ) axillary temperature $>37.5^{\circ} \mathrm{C}$ or rectal or tympanic temperature $>38.0^{\circ} \mathrm{C}$, or history of documented fever within past 24 hours, 4) negative urine pregnancy test, 5) ability to swallow oral medication, 6) availability for follow-up over 42 days, and 7 ) freely provided informed consent. Exclusion criteria were: 1) age <5 years, 2) mixed infection with $P$. vivax), 3) positive urine HCG test for pregnancy, 4) history of epilepsy or psychiatric illness, 5) meeting WHO criteria for severe malaria (2),6) serious co-morbid conditions requiring hospitalization (including, but not limited to severe renal or liver disease, uncontrolled diabetes, systemic bacterial infections), 7) on-going antibiotic therapy, 8) history of hypersensitivity to any of study drug, 9) plans to leave area during next 42 days or to be unavailable for scheduled follow-up, 10) nursing mother, and 11) any other condition which, in the judgment of the study physician would make participation in the study unsafe for the potential volunteer. Eligible, consenting subjects were enrolled; ineligible persons were referred for routine therapy in the clinic.

\section{Randomization and Dosing}

A statistician, otherwise unconnected with the study, randomly distributed cards indicating one of the three regimens into 300 sequentially numbered envelopes, which were sealed and signed. When a subject provided informed consent and enrolled in the study, the envelope corresponding to the subject's study number was opened by a member of the study team. Medications were provided by the Cambodian Ministry of Health as below.

Artesunate-Mefloquine - $12 \mathrm{mg} / \mathrm{kg}$ of artesunate and $25 \mathrm{mg} / \mathrm{kg}$ of mefloquine over 3 days; dosage based on weight up to a maximum dose of $1250 \mathrm{mg}$ mefloquine/ $600 \mathrm{mg}$ artesunate total. One tablet of mefloquine (MQ) contained $250 \mathrm{mg}$ and one tablet of artesunate (AS) contained $50 \mathrm{mg}$.

\begin{tabular}{|lllll|}
\hline Weight (kg) & Age (years) & Day 0 tablets & Day 1 tablets & Day 2 tablets \\
\hline $10-15$ & $5-6$ & $1 \mathrm{AS}$ & $1 \mathrm{MQ}+1 \mathrm{AS}$ & $1 \mathrm{AS}$ \\
\hline $16-25$ & $6-11$ & $1 \mathrm{MQ}+2 \mathrm{AS}$ & $1 \mathrm{MQ}+2 \mathrm{AS}$ & $2 \mathrm{AS}$ \\
\hline $25-35$ & $11-15$ & $1 \mathrm{MQ}+3 \mathrm{AS}$ & $1 \mathrm{MQ}+3 \mathrm{AS}$ & $1 \mathrm{MQ}+3 \mathrm{AS}$ \\
$>35$ & $>15$ & $2 \mathrm{MQ}+4 \mathrm{AS}$ & $2 \mathrm{MQ}+4 \mathrm{AS}$ & $1 \mathrm{MQ}+4 \mathrm{AS}$ \\
\hline
\end{tabular}

Dihydroartemisinin-Piperaquine - One tablet of Duo-Cotecxin (Zhejiang Holley Nanhu Pharmaceutical, Zhejiang, China) containing 40 mg of DHA and 320 mg of piperaquine was administered daily over 3 days. For children under $50 \mathrm{~kg}$, weight-based dosing was used as below from the Cambodian National Center for Parasitology, Entomology and Malaria Control (CNM) 2011 National Treatment Guidelines for Malaria in Cambodia. 


\begin{tabular}{|lllll|}
\hline Weight $\mathbf{( k g )}$ & Age (years) & Day $\mathbf{0}$ (tablets) & Day $\mathbf{1}$ (tablets) & Day 2 (tablets) \\
\hline $10-19$ & 5 & 1 & 1 & 1 \\
\hline $19-30$ & $5-10$ & $1 \frac{1}{2}$ & $1 \frac{1}{2} 2$ & $1 \frac{1}{2}$ \\
\hline $30-40$ & $10-15$ & 2 & 2 & 2 \\
\hline 40 & $>15$ & 3 & 3 & 3 \\
\hline
\end{tabular}

Atovaquone-Proguanil - Adult Malarone tablets containing $250 \mathrm{mg}$ of atovaquone and $100 \mathrm{mg}$ of proguanil hydrochloride were given for three days. For adults $>40 \mathrm{~kg}$ the daily dose was 4 tablets; for subjects $<40 \mathrm{~kg}$ the daily dosage was: $11-20 \mathrm{~kg}, 1$ tablet; $21-30 \mathrm{~kg}, 2$ tablets; $31-40 \mathrm{~kg}, 3$ tablets.

\section{Study Procedures}

Microscopists and laboratory technicians were blinded to the treatments each subject received. Patients were observed in the clinic for one hour after each dose. Before initiating therapy, blood was drawn for in vitro drug resistance assays, resistance markers and genotyping studies. Subjects were asked to re-visit the study unit on days $1,2,3,7,14,18,21,28,35$ and 42 . Subjects were also instructed to return on any other day that that they felt ill in order to monitor clinical recovery/recurrence of malaria symptoms. In the event that a subject failed to re-visit the study unit on schedule, a member of the study team visited the subject. Treatment failures (persistent or recurrent parasitemia) were treated with oral quinine sulfate $10 \mathrm{mg}$ salt/kg three times daily and tetracycline $1 \mathrm{~g}$ daily for 7 days.

\section{Malaria Diagnosis}

Thick and thin blood smears were stained with Giemsa and examined by a certified microscopist using 1000X oil immersion light microscopy. At least 200 ocular fields were examined and the number of asexual and sexual forms per 200 white blood cells (WBC) in the thick smear were recorded separately. This was converted to parasites $/ \mu \mathrm{L}$ for analysis using a conversion multiple of 40 (assuming $8000 \mathrm{WBC} / \mu \mathrm{L}$ ).

In vitro resistance assays

The in vitro drug sensitivity of the $P$. falciparum isolates was assessed by use of a classical isotopic 48-hour test (16). Stock solutions of antimalarial drugs (Institut de Medicine Tropical, Service de santé des armées, Marseille, France) were prepared in methanol and further two-fold serial dilutions in distilled water (Biosedra, France). Two wells of a Falcon 96-well, flat-bottom plate (ATGC, France) were coated with each drug concentration, dried and stored at $4^{\circ} \mathrm{C}$ until use. In vitro testing used reference strains of $P$. falciparum with known drug sensitivities. Blood samples with a parasitemia of at least 6,400 parasites/ $\mu \mathrm{L}$ were washed three times with RPMI 1640 medium (GibcoTM, Invitrogen Corporation, France) by centrifugation (800 g, 10 min, $\left.4^{\circ} \mathrm{C}\right)$ and tested directly without culture adaptation. Infected erythrocytes were suspended (1.5\% hematocrit, 0.1 - 1\% parasitemia) in complete RPMI medium supplemented with $10 \%$ decomplemented human $\mathrm{AB}+$ serum (Biomedia, France) buffered with $25 \mathrm{mM} / \mathrm{L} \mathrm{HEPES}$ and $11 \mathrm{mM} / \mathrm{L} \mathrm{D-(+)-glucose} 25 \mathrm{mM} / \mathrm{L} \mathrm{NaHCO}_{3}$, containing [8-3H] hypoxanthine ( $0.5 \mu \mathrm{Ci} /$ well; Amersham Biosciences, France).

The mixture was distributed $(200 \mu \mathrm{l}$ per well) into 96-well test plates pre-coated with antimalarial drugs. Each plate included two drug-free control wells and one control well without parasites. Plates were incubated for 48 hours at $37^{\circ} \mathrm{C}$ in a $5 \% \mathrm{CO}_{2}$ atmosphere and then the cells were lysed by freeze-thawing. Following collection on glass-fiber filter paper using a cell harvester, the amount of [3H] hypoxanthine incorporated into the parasites' nucleoprotein was determined using a Wallac MicroBeta Trilux counter (Perkin Elmer, France). A log probit approximation was used to determine the $50 \%$ inhibitory concentration $\left(\mathrm{IC}_{50}\right)$, defined as the concentration at which $50 \%$ of $[3 \mathrm{H}]$ hypoxanthine incorporation was inhibited compared to drug-free control wells. The in vitro resistance assay required a starting parasitemia of at least $0.1 \%$ and was not performed on samples with a starting parasitemia $<6,400$ parasites $/ \mu \mathrm{L}(160 \mathrm{parasites} / 200$ WBC).

\section{Parasite Genotyping Studies}

Blood was spotted on \#1 Whatman Filter Paper and stored individually in zip-lock plastic bags with desiccant. Dried blood blots were cut into small pieces, placed in $1.5 \mathrm{ml}$ microcentrifuge tube and lysed in $1 \mathrm{ml}$ of sterile water for 10 minutes at room temperature. During this step, the tubes were vortexed every 1 2 minutes and then centrifuged at 15,000 rpm for 5 minutes. The supernatant was decanted, and DNA precipitate was resuspended in 10 volumes of $5 \%$ chelex-100 (Biorad Laboratories Inc. Hercules, CA), and incubated for 20 minutes at $56^{\circ} \mathrm{C}$ with a brief vortex at high speed before and after incubation. Tubes were then placed in a $100^{\circ} \mathrm{C}$ heating block for 8 minutes and vortexed briefly at high speed after incubation. After a final centrifugation at 15,000 rpm for 2 minutes to precipitate chelex-bound organic material, DNA-containing supernatants were removed by pipette for PCR amplification. Blood spots of about $2 \mathrm{~cm}$ diameters yielded 50 to $100 \mathrm{ng}$ total DNA.

Analysis of amplification of the Pfmdr1 gene was performed as described elsewhere $(16,17)$. Multiplex PCR was used to amplify products from both the Pfmdr1 and $\beta$-tubulin genes in a single tube. PCR was performed in a total volume of $25 \mu \mathrm{L}$ containing PCR buffer, $3.0 \mathrm{mM} \mathrm{MgCl} 2,300 \mu \mathrm{M}$ of each deoxynucleoside triphosphate, and Taq DNA polymerase, $300 \mathrm{nM}$ each Pfmdr1 primer, $100 \mathrm{nM}$ of each $\beta$-tubulin primer, $150 \mathrm{nM}$ of Pfmdr1 probe labeled with 5' Fam and 3' Tamra and $100 \mathrm{nM}$ of $\beta$-tubulin probe labeled with 5' Vic and 3' Tamra. Each assay was performed in duplicate. Reactions were carried out in a Rotorgene real-time PCR system using the following cycling conditions: $1 \times 50^{\circ} \mathrm{C}$ for $2 \mathrm{~min}, 1 \times 95^{\circ} \mathrm{C}$ for $15 \mathrm{~min}$ and 50 cycles of $95^{\circ} \mathrm{C}$ for 15 sec and $60^{\circ} \mathrm{C}$ for 1 minute. Results were accepted as valid if the copy number for control DNA samples were $0.8-1.2$ for 3D7 and $2.8-3.2$ for W2Mef, and if the difference between duplicate copy numbers was $<50 \%$ of the average. The efficiency $E$, was calculated from the slope of a standard curve made from known dilutions of a reference DNA $(E=10-1 /$ slope $)$. The copy number was calculated as: Copy\# $=(E b t * C t b t) /(E m d r * C t m d r)$ where $E b t$ and $E m d r$ are the efficiencies for $\beta$-tubulin and Pfmdr1, respectively, and Ctbt and Ctmdr are the corresponding number of cycles to reach threshold.

Page $4 / 18$ 


\section{Sample Size}

Based on the primary hypothesis that the upper bound of the $95 \%$ confidence interval for the proportion of ACPR in subjects treated with AS/MQ is $<90 \%$ and the lower bound of the $95 \%$ confidence interval for the proportion of ACPR in subjects treated with DHA/PPQ or ATQ/PG is > $90 \%$, approximately 100 subjects per arm was the target sample size.

\section{Statistical Analysis}

The primary analysis was drug efficacy, calculated as the percent of subjects not requiring alternative therapy during the 42-day course of the study and reported with $95 \%$ confidence intervals $(95 \% \mathrm{Cl})$ using the per-protocol dataset per WHO recommendation (13). Additionally, PCR-Corrected Efficacy was estimated by Kaplan-Meier survival analysis and the incidence rates of the arms were compared using the Log-rank test using $\mathrm{R}$ version 4.0 .5 (18) and $\mathrm{R}$ Studio version 1.4.1106 (19) using the following packages: dplyr (version 1.0.5), survival (version 3.2-10), and survminer (version 0.4.9).

\section{Results}

There were 211 subjects meeting criteria for the study who were enrolled from October 2009 - November 2011 and randomized to the AS/MQ (63), DHA/PPQ (77), and ATQ/PG (71) arms as in Figure 2. The target enrollment of 100 subjects per arm was not met due to difficulty recruiting suitable subjects. Overall, 171 of 211 (81.0\%) completed 42-day follow up: 50/63 (79.4\%) in the AS/MQ, 69/77 (89.6\%) in the DHA/PPQ, and 52/71 (73.2\%) in the ATQ/PG arms. Of the subjects excluded from the final analysis, 1 from the AS/MQ arm was excluded due to new $P$. falciparum infection; 6 were excluded from the DHA/PPQ arm due to loss to follow up $(\mathrm{n}=2)$ and $P$. vivax infection $(\mathrm{n}=4)$; and 15 subjects were excluded from the ATQ/PG arm due to allergy $(\mathrm{n}=1)$ and $P$. vivax infection

$(n=14)$. For the primary analysis, $189(89.6 \%)$ were analyzed. Baseline characteristics were similar between treatment groups as in Table 1 without significant differences in demographics, initial parasitemia or clinical presentation. Most subjects were male (79.6\%) with a mean age of 25.8 years (range 5 - 60). Mean temperature was $39.6^{\circ} \mathrm{C}$, mean hematocrit was $40.7 \%$, and the geometric mean parasitemia on enrollment was 11,873 parasites/ $\mu \mathrm{L}$. Baseline gametocytemia was detected in $46.9 \%$ of subjects.

Table 1

Baseline Demographics

\begin{tabular}{|c|c|c|c|c|}
\hline Parameter & AS/MQ & DHA/PPQ & ATQ/PG & $p$ \\
\hline Total, n & 63 & 77 & 71 & \\
\hline Male sex, n (\%) & $50(79)$ & $60(78)$ & $58(82)$ & 0.849 \\
\hline Age, mean (SD), years & $25(13)$ & $26(12)$ & $26(13)$ & 0.720 \\
\hline Weight, mean (SD), kg & $47(14)$ & $49(13)$ & $47(14)$ & 0.605 \\
\hline Duration of symptoms, mean (SD), days & $3(0.5)$ & $3(0.6)$ & $3(0.5)$ & 0.912 \\
\hline Temperature, mean (SD), ${ }^{\circ} \mathrm{C}$ & $39.7(0.9)$ & $39.6(0.9)$ & $39.6(0.7)$ & 0.652 \\
\hline Hematocrit, mean (SD) & $41(6.0)$ & $41(6.5)$ & $40(6.6)$ & 0.573 \\
\hline Parasitemia/ $\mu \mathrm{L}$, geomean (5, 95\%tiles) & $11085(1365,68000)$ & $12364(1468,73200)$ & $12077(1870,69600)$ & 0.848 \\
\hline \multicolumn{5}{|l|}{ Parasite density group, /mL, n (\%) } \\
\hline$\geq 1000$ and $\leq 10,000$ & $34(54)$ & $35(45)$ & $34(48)$ & \multirow[t]{2}{*}{0.594} \\
\hline$>10,000$ and $\leq 100,000$ & $29(46)$ & $42(55)$ & $37(52)$ & \\
\hline Presence of Pf gametocytes, $\mathrm{n}(\%)^{\star}$ & $27(44)$ & $36(47)$ & $35(51)$ & 0.756 \\
\hline
\end{tabular}

Per-protocol PCR-corrected 42-day ACPR rates (95\%Cl) for AS/MQ, DHA/PPQ, and ATQ/PG were 80.6\% (70.8 - 90.5\%), 97.2\% (93.3 - 100\%), and 92.9\% (86.1, 99.6\%), respectively. Kaplan-Meier estimation of 42-day PCR-corrected ACPR (95\%Cl) was 80.8\% (71.6 - 91.2\%) for AS/MQ, 97.3\% (93.6-100\%) for DHA/PPQ, and $94.1 \%$ (88.6-99.9\%) for ATQ/PG (Figure 3). Rates of 42-day ACPR among the 3 arms differed significantly by the Log-Rank test $(p=0.0025)$. Overall day 3 positivity was high at $59.3 \%$, suggesting artemisinin resistance (Table 2 ). 
Table 2

Parasite Clearance

\begin{tabular}{|lllll|}
\hline Day of parasite clearance & $\begin{array}{l}\text { AS/MQ } \\
\mathbf{n}(\%)\end{array}$ & $\begin{array}{l}\text { DHAPPQ } \\
\mathbf{n}(\%)\end{array}$ & $\begin{array}{l}\text { ATQ/PG* } \\
\mathbf{n}(\%)\end{array}$ & $\boldsymbol{p}$ \\
\hline Before day 3 & $15(23.8)$ & $5(6.5)$ & $4(5.8)$ & 0.009 \\
\cline { 1 - 2 } On day 3 & $15(23.8)$ & $24(31.2)$ & $22(31.9)$ & \\
\cline { 1 - 3 } & $33(52.4)$ & $48(62.3)$ & $43(62.3)$ & \\
\hline
\end{tabular}

*1 subject excluded due to drug allergy and another due to day 2 failure in the ATQ/PG arm.

The overall mean Pfmdr1 copy numbers $(95 \% \mathrm{Cl})$ at baseline was $2.2(2.0-2.4$, Table 3$)$. In the AS/MQ arm, treatment failures had higher Pfmdr1 copy numbers than those with ACPR (3.5 versus 1.8 copies, respectively). Interestingly, both DHA/PPQ treatment failures had elevated Pfmdr 1 copy numbers at baseline (3.9 copies), but not at recrudescence (1.0 copies). There was no difference in Pfmdr1 copy numbers between ACPR and failures in the ATQ/PG arm and all recrudescences were wild-type for cytochrome b mutations.

Table 3

Treatment Efficacy and Pfmdr1 Copy Number

\begin{tabular}{|c|c|c|c|c|}
\hline Regimen & Recrudescence & $\begin{array}{l}\text { Number of } \\
\text { Subjects }\end{array}$ & $\begin{array}{l}\text { Pfmdr1 copy number Day } 0 \text { Mean } \\
(95 \% \mathrm{Cl})\end{array}$ & $\begin{array}{l}\text { Pfmdr1 copy number Day of Recrudescence Mean } \\
(95 \% \mathrm{Cl})\end{array}$ \\
\hline \multirow[t]{2}{*}{ AS/MQ } & Yes & $12^{*}$ & $3.5(2.8-4.2)$ & $3.6(2.4-5.3)$ \\
\hline & No & 49 & $1.8(1.6-2.1)$ & \\
\hline \multirow[t]{2}{*}{ DHA/PPQ } & Yes & 2 & 3.9 & 1.0 \\
\hline & No & 74 & $2.2(1.9-2.4)$ & \\
\hline \multirow[t]{2}{*}{ ATQ/PG } & Yes & 4 & 2.1 & 1.6 \\
\hline & No & 65 & $2.2(1.9-2.5)$ & \\
\hline
\end{tabular}

The study was conducted prior to the discovery of currently recognized molecular markers of artemisinin and piperaquine resistance. However, in vitro drug resistance was assessed using a classical isotopic test for samples with $>6,400$ parasites $/ \mu \mathrm{L}$ as in Figure 4 . The only significant difference between groups was observed for $\mathrm{MQ}_{\mathrm{IC}}$, where the $\mathrm{DHA} / \mathrm{PPQ}$ group was marginally higher. Resistance to the artemisinins (AS and DHA) was relatively low based on IC $\mathrm{C}_{50}$ while MQ, CQ and PPQ were all elevated. Mefloquine resistance correlated with increased Pfmdr1 copy number and MQ IC ${ }_{50}$ was higher in those with $>1.5$ copies (Figure 5).

Table 4. Clinical Studies of Uncomplicated Falciparum Malaria in Cambodia, 2001 - 2018 


\begin{tabular}{|c|c|c|c|c|c|c|c|c|c|c|}
\hline Study & Date & Province & Regimen, $n$ & $\begin{array}{l}\text { Baseline } \\
\text { Gametocyte }\end{array}$ & $\begin{array}{l}\text { Day } 3 \\
\text { Positive }\end{array}$ & $\begin{array}{l}\text { Clearance } \\
\text { (hours) }\end{array}$ & PfK13 & PfMdr1 & PfPlasmepsin & $\begin{array}{l}\text { PCR- } \\
\text { ACPR } \\
\text { (days) }\end{array}$ \\
\hline \multirow[t]{14}{*}{$\begin{array}{l}\text { Denis et al., } \\
2006^{a}\end{array}$} & 2001 & Battambang & AS/MQ, 50 & & & & & & & $\begin{array}{l}96 \% \\
\text { PP } \\
(28)\end{array}$ \\
\hline & 2003 & Battambang & AS/MQ, 55 & & & & & & & $\begin{array}{l}92 \% \\
\text { PP } \\
(28)\end{array}$ \\
\hline & 2002 & Pailin & AS/MQ, 81 & & & & & & & $\begin{array}{l}86 \% \\
\text { PP } \\
(28)\end{array}$ \\
\hline & 2004 & Pailin & AS/MQ, 90 & & $10 \%$ & & & $\begin{array}{l}1.5 \\
\text { copies } \\
\text { Overall }\end{array}$ & & $\begin{array}{l}79 \% \\
\text { PP } \\
\text { (42) }\end{array}$ \\
\hline & 2002 & Pursat & AS/MQ, 83 & & & & & & & $\begin{array}{l}93 \% \\
\text { PP } \\
\text { (28) }\end{array}$ \\
\hline & 2004 & Pursat & AS/MQ, 85 & & & & & & & $\begin{array}{l}93 \% \\
\text { PP } \\
\text { (28) }\end{array}$ \\
\hline & 2003 & $\begin{array}{l}\text { Oddar } \\
\text { Meanchey }\end{array}$ & AS/MQ, 92 & & & & & & & $\begin{array}{l}98 \% \\
\text { PP } \\
(28)\end{array}$ \\
\hline & 2002 & $\begin{array}{l}\text { Preah } \\
\text { Vihear }\end{array}$ & AS/MQ, 36 & & & & & & & $\begin{array}{l}97 \% \\
\text { PP } \\
\text { (28) }\end{array}$ \\
\hline & 2004 & $\begin{array}{l}\text { Preah } \\
\text { Vihear }\end{array}$ & AS/MQ, 85 & & & & & & & $\begin{array}{l}100 \% \\
\text { PP } \\
(42)\end{array}$ \\
\hline & 2001 & Kratie & AS/MQ, 50 & & & & & & & $\begin{array}{l}100 \% \\
\text { PP } \\
(28)\end{array}$ \\
\hline & 2003 & Kratie & AS/MQ, 66 & & & & & & & $\begin{array}{l}100 \% \\
\text { PP } \\
(28)\end{array}$ \\
\hline & 2002 & Ratanakiri & AS/MQ, 75 & & & & & & & $\begin{array}{l}100 \% \\
\text { PP } \\
(28)\end{array}$ \\
\hline & 2004 & Ratanakiri & AS/MQ, 80 & & & & & & & $\begin{array}{l}100 \% \\
\text { PP } \\
(42)\end{array}$ \\
\hline & 2003 & $\begin{array}{l}\text { Kampong } \\
\text { Speu }\end{array}$ & AS/MQ, 97 & & & & & & & $\begin{array}{l}97 \% \\
\text { PP } \\
\text { (28) }\end{array}$ \\
\hline \multirow[t]{2}{*}{$\begin{array}{l}\text { Rogers et al., } \\
2009^{b}\end{array}$} & $\begin{array}{l}2006- \\
2008\end{array}$ & Kampot & AS/MQ, 151 & & $11 \%$ & & & $\begin{array}{l}2.7 \\
\text { copies } \\
\text { Fail }\end{array}$ & & $\begin{array}{l}81 \% \\
\text { KM } \\
(42)\end{array}$ \\
\hline & & & & & & & & $\begin{array}{l}1.9 \\
\text { copies } \\
\text { ACPR }\end{array}$ & & \\
\hline \multirow{2}{*}{$\begin{array}{l}\text { Noedl et al., } \\
2008 \\
\text { Noedl et al., } \\
2010^{c}\end{array}$} & $\begin{array}{l}2006- \\
2007\end{array}$ & Battambang & AS, 74 & & $22 \%$ & $\mathrm{PCT}=58$ & & $\begin{array}{l}1.1 \\
\text { copies } \\
\text { Overall }\end{array}$ & & $\begin{array}{l}94 \% \\
\text { KM } \\
(28)\end{array}$ \\
\hline & & & QN+TET, 37 & & $34 \%$ & $\mathrm{PCT}=78$ & & & & $\begin{array}{l}100 \% \\
\text { KM } \\
(28)\end{array}$ \\
\hline \multirow[t]{2}{*}{$\begin{array}{l}\text { Rueangweerayut } \\
\text { et al., } 2012^{\mathrm{d}}\end{array}$} & $\begin{array}{l}2007- \\
2008\end{array}$ & Pailin & AS/PYR, 140 & & $37 \%$ & PCT $=64$ & & & & $\begin{array}{l}90 \% \\
\text { KM } \\
(42)\end{array}$ \\
\hline & & & AS/MQ, 71 & & $38 \%$ & $\mathrm{PCT}=64$ & & & & $\begin{array}{l}100 \% \\
\mathrm{KM} \\
(42)\end{array}$ \\
\hline \multirow[t]{2}{*}{$\begin{array}{l}\text { Dondorp et al., } \\
200 \mathrm{e}^{\mathrm{e}}\end{array}$} & $\begin{array}{l}2007- \\
2008\end{array}$ & Pailin & AS/MQ, 20 & $30 \%$ & $55 \%$ & $\mathrm{PCT}=72$ & & $\begin{array}{l}5 \% \\
\text { amplified }\end{array}$ & & $\begin{array}{l}95 \% \\
\text { ITT } \\
(63)\end{array}$ \\
\hline & & & AS, 20 & $\begin{array}{l}25 \% \\
\quad \text { Page } 7 / 1\end{array}$ & & $\mathrm{PCT}=84$ & & & & $70 \%$ \\
\hline
\end{tabular}




\begin{tabular}{|c|c|c|c|c|c|c|c|c|c|}
\hline \multirow[t]{8}{*}{$\begin{array}{l}\text { Leang et al., } \\
2013^{f}\end{array}$} & $\begin{array}{l}2008- \\
2009\end{array}$ & Pailin & DHA/PPQ, 59 & & $26 \%$ & & & $\begin{array}{l}17 \% \\
\text { amplified }\end{array}$ & $\begin{array}{l}90 \% \\
\text { KM } \\
(42)\end{array}$ \\
\hline & $\begin{array}{l}2009- \\
2010\end{array}$ & Pailin & DHA/PPQ, 41 & & $33 \%$ & & & $\begin{array}{l}24 \% \\
\text { amplified }\end{array}$ & $\begin{array}{l}92 \% \\
\text { KM } \\
(42)\end{array}$ \\
\hline & $\begin{array}{l}2010- \\
2011\end{array}$ & Pailin & DHA/PPQ, 30 & & $45 \%$ & & & $\begin{array}{l}16 \% \\
\text { amplified }\end{array}$ & $\begin{array}{l}76 \% \\
\text { KM } \\
(42)\end{array}$ \\
\hline & 2008 & Pursat & DHA/PPQ, 86 & & $8 \%$ & & & $\begin{array}{l}32 \% \\
\text { amplified }\end{array}$ & $\begin{array}{l}99 \% \\
\text { KM } \\
(42)\end{array}$ \\
\hline & 2010 & Pursat & DHA/PPQ, 64 & & $10 \%$ & & & $\begin{array}{l}25 \% \\
\text { amplified }\end{array}$ & $\begin{array}{l}90 \% \\
\text { KM } \\
(42)\end{array}$ \\
\hline & 2009 & $\begin{array}{l}\text { Preah } \\
\text { Vihear }\end{array}$ & DHA/PPQ, 60 & & $5 \%$ & & & & $\begin{array}{l}100 \% \\
\text { KM } \\
(42)\end{array}$ \\
\hline & $\begin{array}{l}2009- \\
2010\end{array}$ & Ratanakiri & DHA/PPQ, 59 & & $0 \%$ & & & & $\begin{array}{l}100 \% \\
\text { KM } \\
(42)\end{array}$ \\
\hline & 2010 & Ratanakiri & DHA/PPQ, 61 & & $0 \%$ & & & & $\begin{array}{l}100 \% \\
\text { KM } \\
(42)\end{array}$ \\
\hline \multirow[t]{3}{*}{$\begin{array}{l}\text { Bethell et al., } \\
2011^{\mathrm{g}}\end{array}$} & $\begin{array}{l}2008- \\
2009\end{array}$ & Battambang & AS2, 75 & $13 \%$ & $49 \%$ & PCT $=74$ & & & $\begin{array}{l}88 \% \\
\text { PP } \\
(42)\end{array}$ \\
\hline & & & AS4, 40 & $10 \%$ & $46 \%$ & PCT $=78$ & & & $\begin{array}{l}90 \% \\
\mathrm{PP} \\
(42)\end{array}$ \\
\hline & & & AS6, 28 & $21 \%$ & $48 \%$ & $\mathrm{PCT}=78$ & & & $\begin{array}{l}79 \% \\
\text { PP } \\
\text { (42) }\end{array}$ \\
\hline \multirow[t]{2}{*}{$\begin{array}{l}\text { Amaratunga et } \\
\text { al., } 2012^{\mathrm{h}}\end{array}$} & $\begin{array}{l}2009- \\
2010\end{array}$ & Pursat & AS/MQ, 180 & $16 \%$ & & $\begin{array}{l}\mathrm{PC}_{1 / 2}= \\
5.9\end{array}$ & & & \\
\hline & & & & & & PCT = 78 & & & \\
\hline \multirow[t]{6}{*}{ This Study } & $\begin{array}{l}2009- \\
2011\end{array}$ & $\begin{array}{l}\text { Oddar } \\
\text { Meanchey }\end{array}$ & AS/MQ, 63 & $44 \%$ & $52 \%$ & & & $\begin{array}{l}3.5 \\
\text { copies } \\
\text { Fail }\end{array}$ & $\begin{array}{l}81 \% \\
\text { KM } \\
(42)\end{array}$ \\
\hline & & & & & & & & $\begin{array}{l}1.8 \\
\text { copies } \\
\text { ACPR }\end{array}$ & \\
\hline & & & DHA/PPQ, 77 & $47 \%$ & $62 \%$ & & & $\begin{array}{l}3.9 \\
\text { copies } \\
\text { Fail }\end{array}$ & $\begin{array}{l}97 \% \\
\text { KM } \\
(42)\end{array}$ \\
\hline & & & & & & & & $\begin{array}{l}2.2 \\
\text { copies } \\
\text { ACPR }\end{array}$ & \\
\hline & & & ATQ/PG, 71 & $51 \%$ & $62 \%$ & & & $\begin{array}{l}2.1 \\
\text { copies } \\
\text { Fail }\end{array}$ & $\begin{array}{l}94 \% \\
\mathrm{KM} \\
(42)\end{array}$ \\
\hline & & & & & & & & $\begin{array}{l}2.2 \\
\text { copies } \\
\text { ACPR }\end{array}$ & \\
\hline \multirow[t]{2}{*}{ Lon et al., 2014 } & $\begin{array}{l}2010- \\
2011\end{array}$ & $\begin{array}{l}\text { Oddar } \\
\text { Meanchey }\end{array}$ & $\mathrm{DHA} / \mathrm{PPQ} \times 2,8$ & $13 \%$ & $50 \%$ & PCT $=80$ & $\begin{array}{l}82 \% \\
\mathrm{C} 580 \mathrm{Y}\end{array}$ & $\begin{array}{l}1.2 \\
\text { copies } \\
\text { Fail }\end{array}$ & $\begin{array}{l}75 \% \\
\text { PP } \\
(42)\end{array}$ \\
\hline & & & DHA/PPQ x3, 12 & $42 \%$ & $42 \%$ & PCT $=68$ & & $\begin{array}{l}1.1 \\
\text { copies } \\
\text { ACPR }\end{array}$ & $\begin{array}{l}82 \% \\
\text { PP } \\
(42)\end{array}$ \\
\hline \multirow[t]{2}{*}{$\begin{array}{l}\text { Leang et al., } \\
2015^{\mathrm{k}}\end{array}$} & $\begin{array}{l}2011- \\
2013\end{array}$ & West & DHA/PPQ, 147 & & $31 \%$ & & $\begin{array}{l}87 \% \\
\mathrm{C} 580 \mathrm{Y}\end{array}$ & $\begin{array}{l}16 \% \\
\text { amplified }\end{array}$ & $\begin{array}{l}85 \% \\
\text { PP } \\
\text { (42) }\end{array}$ \\
\hline & & East & DHA/PPQ, 278 & & $17 \%$ & & $23 \%$ & $21 \%$ & $98 \%$ \\
\hline
\end{tabular}




\begin{tabular}{|c|c|c|c|c|c|c|c|c|c|c|}
\hline & & & & & & & C580Y & amplified & & $\begin{array}{l}\mathrm{PP} \\
(42)\end{array}$ \\
\hline \multirow[t]{4}{*}{$\begin{array}{l}\text { Ashley et al., } \\
2014\end{array}$} & \multirow[t]{4}{*}{$\begin{array}{l}2011- \\
2013\end{array}$} & Pailin & $\begin{array}{l}\text { AS4+DHA/PPQ, } \\
100\end{array}$ & $19 \%$ & $66 \%$ & $\begin{array}{l}\mathrm{PC}_{1 / 2}= \\
6.1\end{array}$ & \multirow[t]{2}{*}{$\begin{array}{l}\sim 75 \% \\
\mathrm{C} 580 Y\end{array}$} & & \multirow[t]{2}{*}{$\sim 50 \%$} & \multirow[t]{2}{*}{$\begin{array}{l}98 \% \\
\mathrm{PP} \\
(42)\end{array}$} \\
\hline & & Pursat & $\begin{array}{l}\text { AS4+DHA/PPQ, } \\
120\end{array}$ & $18 \%$ & $60 \%$ & $\begin{array}{l}\mathrm{PC}_{1 / 2}= \\
5.6\end{array}$ & & & & \\
\hline & & $\begin{array}{l}\text { Preah } \\
\text { Vihear }\end{array}$ & $\begin{array}{l}\text { AS2/4+DHA/PPQ, } \\
120\end{array}$ & $5 \%$ & $18 \%$ & $\begin{array}{l}\mathrm{PC}_{1 / 2}= \\
3.0\end{array}$ & Diverse & & Mostly WT & \\
\hline & & Ratanakiri & $\begin{array}{l}\text { AS2/4+DHA/PPQ, } \\
120\end{array}$ & $6 \%$ & $7 \%$ & $\begin{array}{l}\mathrm{PC}_{1 / 2}= \\
3.0\end{array}$ & $\begin{array}{l}\text { Mostly } \\
\text { WT }\end{array}$ & & $100 \% \mathrm{WT}$ & \\
\hline \multirow[t]{3}{*}{$\begin{array}{l}\text { Amaratunga et } \\
\text { al., } 2016^{\mathrm{m}}\end{array}$} & \multirow[t]{3}{*}{$\begin{array}{l}2012- \\
2013\end{array}$} & Pursat & DHA/PPQ, 110 & $17 \%$ & $61 \%$ & $\begin{array}{l}\mathrm{PC}_{1 / 2}= \\
6.1\end{array}$ & $\begin{array}{l}77 \% \\
\text { Mutant }\end{array}$ & $\begin{array}{l}0 \% \\
\text { amplified } \\
\text { Fail }\end{array}$ & & $\begin{array}{l}63 \% \\
\mathrm{KM} \\
(63)\end{array}$ \\
\hline & & $\begin{array}{l}\text { Preah } \\
\text { Vihear }\end{array}$ & DHA/PPQ, 65 & $9 \%$ & $26 \%$ & $\begin{array}{l}\mathrm{PC}_{1 / 2}= \\
3.0\end{array}$ & $\begin{array}{l}34 \% \\
\text { Mutant }\end{array}$ & $\begin{array}{l}11 \% \\
\text { amplified } \\
\text { ACPR }\end{array}$ & & $\begin{array}{l}85 \% \\
\mathrm{KM} \\
(63)\end{array}$ \\
\hline & & Ratanakiri & DHA/PPQ, 66 & $2 \%$ & $3 \%$ & $\begin{array}{l}\mathrm{PC}_{1 / 2}= \\
2.4\end{array}$ & $\begin{array}{l}11 \% \\
\text { Mutant }\end{array}$ & & & $\begin{array}{l}98 \% \\
\mathrm{KM} \\
(63)\end{array}$ \\
\hline \multirow{2}{*}{$\begin{array}{l}\text { Saunders et al., } \\
2014 \\
\text { Spring et al., } \\
2015^{\mathrm{n}}\end{array}$} & \multirow[t]{2}{*}{$\begin{array}{l}2012- \\
2014\end{array}$} & \multirow[t]{2}{*}{$\begin{array}{l}\text { Oddar } \\
\text { Meanchey }\end{array}$} & DHA/PPQ, 51 & $10 \%$ & $65 \%$ & \multirow{2}{*}{$\begin{array}{l}\mathrm{PC}_{1 / 2}= \\
6.4 \\
\mathrm{PCT}=80\end{array}$} & \multirow{2}{*}{$\begin{array}{l}65 \% \\
\text { C580Y } \\
\\
31 \% \\
\text { R539T }\end{array}$} & & & $\begin{array}{l}58 \% \\
\text { mITT } \\
(42)\end{array}$ \\
\hline & & & $\mathrm{DHA} / \mathrm{PPQ}+\mathrm{PQ}, 50$ & $8 \%$ & $42 \%$ & & & & & $\begin{array}{l}50 \% \\
\text { mlTT } \\
(42)\end{array}$ \\
\hline \multirow[t]{3}{*}{$\begin{array}{l}\text { Leang et al., } \\
2016^{\circ}\end{array}$} & \multirow[t]{3}{*}{$\begin{array}{l}2014- \\
2015\end{array}$} & Pailin & AS/PYR, 55 & $0 \%$ & $13 \%$ & & \multirow[t]{3}{*}{$\begin{array}{l}96 \% \\
\text { C580Y }\end{array}$} & & & $\begin{array}{l}84 \% \\
\mathrm{KM} \\
(42)\end{array}$ \\
\hline & & Pursat & AS/PYR, 60 & $8 \%$ & $44 \%$ & & & & & $\begin{array}{l}90 \% \\
\mathrm{KM} \\
(42)\end{array}$ \\
\hline & & Battambang & AS/PYR, 8 & $0 \%$ & $25 \%$ & & & & & $\begin{array}{l}100 \% \\
\mathrm{KM} \\
(42)\end{array}$ \\
\hline \multirow[t]{4}{*}{$\begin{array}{l}\text { Wojnarski et al., } \\
2019^{p}\end{array}$} & \multirow[t]{4}{*}{$\begin{array}{l}2014- \\
2015\end{array}$} & \multirow[t]{2}{*}{$\begin{array}{l}\text { Oddar } \\
\text { Meanchey }\end{array}$} & ATQ/PG+PQ, 79 & $21 \%$ & $42 \%$ & $\begin{array}{l}\mathrm{PC}_{1 / 2}= \\
5.6\end{array}$ & $\begin{array}{l}100 \% \\
\mathrm{C} 580 \mathrm{Y}\end{array}$ & & & $\begin{array}{l}88 \% \\
\mathrm{KM} \\
(42)\end{array}$ \\
\hline & & & $\begin{array}{l}\text { AS+ATQ/PG+PQ, } \\
78\end{array}$ & & $42 \%$ & $\begin{array}{l}\mathrm{PC}_{1 / 2}= \\
5.9\end{array}$ & $\begin{array}{l}99 \% \\
\text { C580Y }\end{array}$ & & & $\begin{array}{l}91 \% \\
\mathrm{KM} \\
(42)\end{array}$ \\
\hline & & \multirow[t]{2}{*}{ Kratie } & ATQ/PG+PQ, 24 & $19 \%$ & $17 \%$ & $\begin{array}{l}\mathrm{PC}_{1 / 2}= \\
5.6\end{array}$ & $\begin{array}{l}91 \% \\
\text { C580Y }\end{array}$ & & & $\begin{array}{l}100 \% \\
\mathrm{KM} \\
(42)\end{array}$ \\
\hline & & & $\begin{array}{l}\text { AS+ATQ/PG+PQ, } \\
24\end{array}$ & & $4 \%$ & $\begin{array}{l}\mathrm{PC}_{1 / 2}= \\
5.3\end{array}$ & $\begin{array}{l}86 \% \\
\text { C580Y }\end{array}$ & & & $\begin{array}{l}94 \% \\
\mathrm{KM} \\
(42)\end{array}$ \\
\hline \multirow{6}{*}{$\begin{array}{l}\text { Van der Pluijm } \\
\text { et al., } 2019 \\
\text { Van der Pluijm } \\
\text { et al., } 2020^{q}\end{array}$} & \multirow[t]{6}{*}{$\begin{array}{l}2015- \\
2018\end{array}$} & \multirow[t]{4}{*}{ Pailin } & $\mathrm{DHA} / \mathrm{PPQ}+\mathrm{PQ}, 9$ & \multirow[t]{6}{*}{$41 \%$} & \multirow[t]{4}{*}{$72 \%$} & \multirow[t]{4}{*}{$\begin{array}{l}\mathrm{PC}_{1 / 2}= \\
7.2\end{array}$} & \multirow[t]{4}{*}{$\begin{array}{l}97 \% \\
\text { C580Y }\end{array}$} & \multirow[t]{6}{*}{$\begin{array}{l}0 \% \\
\text { amplified }\end{array}$} & \multirow[t]{4}{*}{$69 \%$} & $\begin{array}{l}52 \% \\
\mathrm{KM} \\
(42)\end{array}$ \\
\hline & & & $\begin{array}{l}\mathrm{DHA} / \mathrm{PPQ}+\mathrm{MQ}+\mathrm{PQ} \\
11\end{array}$ & & & & & & & $\begin{array}{l}100 \% \\
\mathrm{KM} \\
(42)\end{array}$ \\
\hline & & & $\mathrm{AS} / \mathrm{MQ}+\mathrm{PQ}, 22$ & & & & & & & $\begin{array}{l}100 \% \\
\mathrm{KM} \\
(42)\end{array}$ \\
\hline & & & $\begin{array}{l}\mathrm{DHA} / \mathrm{PPQ}+\mathrm{MQ}+\mathrm{PQ} \\
22\end{array}$ & & & & & & & $\begin{array}{l}100 \% \\
\text { KM } \\
(42)\end{array}$ \\
\hline & & \multirow[t]{2}{*}{ Pursat } & DHA/PPQ+PQ, 8 & & $46 \%$ & $\begin{array}{l}\mathrm{PC}_{1 / 2}= \\
6.3\end{array}$ & $\begin{array}{l}89 \% \\
\text { C580Y }\end{array}$ & & $79 \%$ & $\begin{array}{l}25 \% \\
\mathrm{KM} \\
(42)\end{array}$ \\
\hline & & & $\begin{array}{l}\mathrm{DHA} / \mathrm{PPQ}+\mathrm{MQ}+\mathrm{PQ} \\
11\end{array}$ & & & & & & & $\begin{array}{l}100 \% \\
\mathrm{KM} \\
(42)\end{array}$ \\
\hline
\end{tabular}




\begin{tabular}{|c|c|c|c|c|c|}
\hline \multirow[t]{2}{*}{$\begin{array}{l}\text { Preah } \\
\text { Vihear }\end{array}$} & $\mathrm{AS} / \mathrm{MQ}+\mathrm{PQ}, 3$ & & $14 \%$ & \multirow[t]{2}{*}{$\begin{array}{l}\mathrm{PC}_{1 / 2}= \\
5.2\end{array}$} & \multirow[t]{2}{*}{$\begin{array}{l}100 \% \\
\text { C580Y }\end{array}$} \\
\hline & $\begin{array}{l}\mathrm{DHA} / \mathrm{PPQ}+\mathrm{MQ}+\mathrm{PQ}, \\
4\end{array}$ & & & & \\
\hline \multirow[t]{2}{*}{ Ratanakiri } & $\mathrm{DHA} / \mathrm{PPQ}+\mathrm{PQ}, 44$ & $16 \%$ & $52 \%$ & $\begin{array}{l}\mathrm{PC}_{1 / 2}= \\
7.0\end{array}$ & $\begin{array}{l}85 \% \\
\mathrm{C} 580 \mathrm{Y}\end{array}$ \\
\hline & $\begin{array}{l}\mathrm{DHA} / \mathrm{PPQ}+\mathrm{MQ}+\mathrm{PQ}, \\
46\end{array}$ & & & & \\
\hline
\end{tabular}

Abbreviations: PP, Per-Protocol; QN, Quinine; TET, Tetracycline; PYR, Pyronaridine; PQ, Primaquine; PCT, Parasite Clearance Time; PC $1 / 2$, Parasite Clearance Half-Life; WT, Wild-type; ITT, Intention-to-Treat; mITT, Modified Intention-to-Treat; KM, Kaplan-Meier

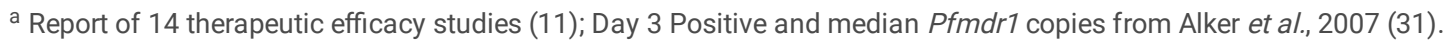

b Therapeutic efficacy study reporting mean Pfmdr1 copies; Failures had 3.6 copies at recrudescence (12).

${ }^{\mathrm{c}}$ Randomized control trial reporting median PCT and Pfmdr1 copies $(55,56)$.

${ }^{d}$ Phase 3, randomized control trial reporting median PCT (57).

${ }^{\mathrm{e}}$ Randomized control trial with another site in Wang Pha, Thailand; reporting median PCT (32).

${ }^{f}$ Report of 8 therapeutic efficacy studies (33).

${ }^{g}$ Randomized control trial comparing 2, 4, and 6 mg/kg AS; reporting median PCT (58).

${ }^{\mathrm{h}}$ Parasite clearance rate study reporting geometric mean $\mathrm{PC}_{1 / 2}(23)$.

${ }^{i}$ Randomized control trial; mean Pfmdr1 copies at recrudescence were 3.6, 1.0, and 1.6 for AS/MQ, DHA/PPQ, and ATQ/PG, respectively.

j Randomized control trial comparing 2- versus 3-day regimens of DHA/PPQ, reporting median PCT and Pfmdr1 (38); PfK13 mutations were essentially at fixation (25).

${ }^{k}$ Therapeutic efficacy study; Western refers to Battambang, Pursat, Kampot, and Kampong Speu; Eastern refers to Kratie, Preah Vihear, Ratanakiri, and Kampong Thom (9).

I TRACl: Parasite clearance rate study of AS 2 or $4 \mathrm{mg} / \mathrm{kg}$ with DHA/PPQ, reporting median $\mathrm{PC}_{1 / 2}$ (26); PfK13 and Pfplasmepsin status reported in van der Pluijm et al., 2019 (34).

$\mathrm{m}$ Therapeutic efficacy study reporting median $\mathrm{PC}_{1 / 2}(27)$.

${ }^{\mathrm{n}}$ Randomized control trial reporting median $\mathrm{PC}_{1 / 2}$ and PCT $(8,25)$; Gametocytemia reported in Lin et al., 2017 (52).

${ }^{\circ}$ Therapeutic efficacy study (59).

p Randomized control trial reporting median $\mathrm{PC}_{1 / 2}(42)$.

q TRACII: Randomized control trial; Gametocytemia for Pailin and Pursat refer to the 17 subjects receiving DHA/PPQ $(34,35)$.

\section{Discussion}

In this 2009 - 2011 therapeutic efficacy study in Oddar Meanchey, PCR-corrected failures rates for AS/MQ, ATQ/PG and DHA/PPQ were 19\%, 6\%, and 3\%, respectively. The overall Day 3 Positivity was nearly $60 \%$, suggesting a high level of clinical artemisinin resistance. However in vitro parasite resistance assays on a subset of samples did not indicate significant artemisinin resistance. The WHO recommends changing therapy when failure rates exceed $10 \%$ and instituting containment measures when Day 3 positivity exceeds $10 \%(20)$. Data from the study was provided to CNM at the time and used to support changes to therapy including the transition to DHA/PPQ. Overall, there were 2.2 copies of Pfmdr1 on average, consistent with previous work by NAMRU-2 which estimated mean copy number at this site at $\sim 2$. Further, there was both in vitro evidence of $\mathrm{MQ}$ resistance and unacceptably high rates of $\mathrm{AS} / \mathrm{MQ}$ clinical 
failures at this site. Treatment failures in the AS/MQ arm had higher baseline Pfmdr1 copy numbers than those with ACPR (3.5 versus 1.8 copies, respectively). Both DHA/PPQ treatment failures had baseline Pfmdr1 amplification, but lost amplification at recrudescence. Baseline gametocytemia, important for mosquito transmission, was the highest reported among clinical studies in Cambodia (47\%) and higher than the global average of $12 \%$ in a meta-analysis (21). Some factors predictive of baseline gametocytemia in Asia were present in this study (age, male gender, and low density of parasitemia) but not others (afebrile disease and anemia) (21). This also supports the notion of clinically smoldering infections in the present study indicative of partially treated and/or resistant infections.

This study occurred during the national transition from AS/MQ and helps bridge the historic gap in understanding the transition to full-blown DHA/PPQ failures that occurred rapidly between 2008 - 2014 on the Thai-Cambodian border. Table 4 summarizes clinical studies of uncomplicated Falciparum malaria in Cambodia from 2001-2018. A brief review of historic and interim findings related to resistance to the drugs used in the present study is incorporated with a discussion of the findings. While some of the commonly used molecular markers of resistance used today were not available, there was clear elevation of Pfmdr1 copy number among subjects with $\mathrm{AS} / \mathrm{MQ}$ failures, as well as elevated MQ $\mathrm{IC}_{50} \mathrm{~s}$ in those with elevated copy numbers.

[Table 4]

\section{Artemisinin Resistance}

Artemisinin resistance is defined by Parasite Clearance Half-Life $\left(\mathrm{PC}_{1 / 2}\right) \geq 5$ hours, but Day 3 Positivity $\geq 10 \%$ is considered a useful clinical surrogate (22). Day 3 positivity increased rapidly (10 to 55\%) in Pailin in AS/MQ studies from $2004-2008$. In DHA/PPQ studies from 2008 onward, Day 3 positivity was generally higher in Western than Eastern provinces. In that period, the rate in Pailin increased from $26-72 \%$ while remaining relatively constant (between 42 and 65\%) in Oddar Meanchey. Parasite Clearance Half-Life was 5.9 hours in a 2009 - 2010 AS/MQ parasite clearance rate study in Pursat (23) and was generally longer in Western than Eastern Cambodia. Since 2014, average $\mathrm{PC}_{1 / 2}$ has consistently exceeded 5 hours throughout Cambodia.

Mutations in PfKelch 13 (K13) are newer molecular markers of artemisinin resistance (24) and with the exception of Eastern Cambodia, have essentially been at fixation since 2010. The C580Y single nucleotide polymorphism (SNP) was higher in Western than Eastern provinces, but became the dominant K13 allele throughout Cambodia by 2014 - 2015. In a 2010 Oddar Meanchey study, all failures were C580Y, and 3 year later, all failures were either C580Y or R539T (25). Several K13 mutations were strongly associated with prolonged $\mathrm{PC}_{1 / 2}$ in Pailin and with recrudescence throughout Cambodia (26, 27). Near simultaneous studies found $\mathrm{C} 580 \mathrm{Y}$ associated with high Day 3 positivity, prolonged $\mathrm{PC}_{1 / 2}$, and post-treatment gametocytemia in Oddar Meanchey (25) and with treatment failures throughout Cambodia (9). In this 2009 - 2011 study, we observed Day 3 positivity ranging from 51-62\% suggesting a level of artemisinin resistance not previously appreciated at the time in Northern Cambodia. Therefore, the difference in efficacy between AS/MQ and DHA/PPQ (79\% and 97\%, respectively) probably reflects differing partner drug susceptibility. Resistance to AS and DHA as measured by $\mathrm{IC}_{50}$ was relatively low, though it is unclear how predictive these were in the isotopic assays used at the time. In the interval, ring-stage survival assays for the artemisinins have been developed and are more widely used (28).

\section{Mefloquine Resistance}

Mefloquine resistance was reported shortly after its introduction on the Thai-Cambodian border (29). Amplification of Pfmdr1 is a molecular marker of MQ resistance, which leads to higher expression of the Multidrug Resistance Protein 1 (MDR1) pump and higher MQ IC ${ }_{50}(14,30)$. An early AS/MQ efficacy study found an association between high copy numbers and treatment failures (31) and another efficacy study reported a rise in copy numbers in recrudescent compared to baseline samples (3.6 versus 2.7, respectively) (12). However, a later AS/MQ randomized study found no association with Pfmdr1 copy numbers and treatment failure (32). During the transition to DHA/PPA, therapeutic efficacy studies from $2008-2010$ surprisingly revealed increasing MQ IC ${ }_{50}$, though 17 of 18 failures were Pfmdr1 deamplified (33). This discrepancy was seemingly resolved in $2012-2013$ efficacy studies in 3 provinces where all 48 failures were Pfmdr1 deamplified and had lower MQ IC 50 than those with ACPR (27). These studies suggest that PPQ exposure drives Pfmdr1 deamplification but additional factors may play a role in MQ resistance. By the time of the 2015 - 2018 Tracking Resistance to Artemisinin Collaboration 2 (TRACII) study, no isolates were Pfmdr1 amplified following the prior withdrawal of AS/MQ as first line agent for several years (34, 35). In the present study, we observed high baseline Pfmdr1 copy numbers, especially among AS/MQ failures. Both DHA/PPQ failures had deamplified copy numbers at recrudescence (from 3.9 to 1 copies), suggesting either selection of a minor population or de novo deamplification after exposure to PPQ.

\section{Piperaquine Resistance}

Piperaquine, a bisquinoline similar to chloroquine was used as monotherapy in Pailin and elsewhere for long periods since the 1990s (33). Piperaquine resistance appears to be multifactorial with multiple molecular markers identified and confirmed clinically over the past decade. The mutant $P f c r t$ haplotype CVIET, associated with CQ and PPQ resistance, likely originated in Southeast Asia in the 1970s or earlier (36, 37). All tested isolates in the $2008-2010$ therapeutic efficacy studies and the 2010 - 2011 Oddar Meanchey study were CVIET $(33,38)$. Deamplification of Pfmdr 1 is another marker of PPQ resistance, seen in 17 of 18 failures in a study from 2008 - 2010 and all failures in a 2011 - 2013 study $(9,33)$. The former study did not see a rise in PPQ IC ${ }_{50}$ over time and the latter study did not correlate copy numbers to clinical outcomes. Significant clinical failure of DHA/PPQ was first reported in a 2012 - 2014 Oddar Meanchey study that was halted (8). An ex vivo susceptibility study determined that DHA/PPQ clinical failures were preceded by increases in PPQ IC ${ }_{90}$ and decreases in Pfmdr1 copy number and MQ IC ${ }_{50}$ (39). By 2012, a study found that recrudescent parasites had higher PPQ IC ${ }_{50}$ and lower MQ IC 50 than those with ACPR; none of the failures were Pfmdr1 amplified (27). At the onset of the $2015-2018$ TRACll study, where all isolates were Pfmdr1 deamplified, DHA/PPQ was still utilized (34). That study confirmed high rates of failure of DHA/PPQ, associated with Pfplasmepsin 2/3 amplification and 4 new Pfcrt mutations (T93S, H97Y, F145I, I218F) (34). Retrospective analysis of the 2011 - 2013 TRACl study found that over half of isolates in Western Cambodia were Pfplasmepsin 2/3 amplified. At the time, these were mutations were rarely found in the East, but have since expanded to be dominant throughout Cambodia 
as observed in the TRACII study (34). The present study had only 2 DHA/PPQ failures, but to our knowledge, deamplification of Pfmdr 1 in paired baseline and recrudescent samples has not been previously described.

\section{Counterbalance of PfMDR1 and PfCRT mutations}

The differential binding of quinoline drugs and opposing actions of the MDR1 and Chloroquine Resistance Transporter (CRT) pumps affect parasite susceptibility to antimalarials (40). The CRT pump transports CQ out of the digestive vacuole, where it interferes with heme detoxification; the MDR1 pump transports MQ into the digestive vacuole and away from a cytosolic site of action (40). Chloroquine resistance arose along the Thai-Cambodian border in the late 1950s (5), associated with the mutant Pfcrt haplotype CVIET (codons 72 - 76) (36). A genetic engineering study demonstrated that Pfmdr1 haplotypes modulate susceptibilities to different antimalarials in the presence of CVIET (40). The Pfmdr1 NF haplotype (wild-type N86, mutant Y184F) decreases susceptibility to CQ and PPQ in presence of Pfcrt CVIET (40) and is associated with MQ resistance (31). The Pfmdr1 NY haplotype (wild-type for both N86 and Y184) is associated with MQ sensitivity (31). In a retrospective analysis of a 2004 AS/MQ Pailin study, most parasites were either Pfmdr1 NF(76\%) or NY (17\%) haplotypes (31). Both Pfmdr1 haplotypes also co-occurred with Pfmdr1 amplification, though they were more common among NY isolates (40). From 2009 - 2013, coinciding with the transition to DHA/PPQ, northern Cambodian isolates saw declining Pfmdr1 amplification and increasing Y184F mutations (7). In another Oddar Meanchey study done around the same time as this study, all isolates were CVIET and 19 of 20 were Pfmdr1 NF and mostly deamplified (38). Not surprisingly, the mean $C Q I_{50}$ in this study was above $85 \mathrm{nM}$, suggesting baseline CQ resistance.

\section{Atovaquone-Proguanil Resistance}

Since 2006, ATQ/PG has been used in WHO-sponsored containment efforts along the Thai border and remains effective in preventing and treating multidrug resistant Falciparum $(41,42)$. Due to its primary activity against the causal liver stage $(43)$, resistance could quickly develop when used against the bloodstage or for mass drug administration (44). Atovaquone inhibits falciparum cytochrome bc ${ }_{1}$ complex and 3 cytochrome b (Pfcytb) SNPs (Y268N, Y268S, $\mathrm{Y} 268 \mathrm{C})$ are strongly associated with in vitro resistance and late clinical failure $(41,43)$. Though mutations in Pfcytb appear to occur frequently, they may come at a fitness cost and the inability to persist in the population (41). Recent studies in Oddar Meanchey suggest a low level of ATQ resistance. All failures in the 2012 - 2014 DHA/PPQ Oddar Meanchey study were wild-type Pfcytb 268 and sensitive to ATQ in vitro (43). All parasites in a 2014 - 2015 Oddar Meanchey and Kratie study were Pfcytb wild-type at baseline, but one late failure developed the Y268C mutation on day 28 (45). The partner drug, proguanil, is biotransformed into the dihydrofolate reductase inhibitor cycloguanil (CYC) by CYP2C19. It is believed that PG has greater synergy with ATQ (46, 47). One reason is that a high level of CYC resistance has been long established in Southeast Asia, likely mediated by the Pfdhfr SNPs S108T and A16V (42, 45, 47). Another reason is the sizeable portion of Asians who may be CYP2C19 poor metabolizers, which result in lower CYC levels (41, 47). Interestingly, while all 14 failures in the 2014 - 2015 study lacked Pfdhfr S108T and A16V mutations, they were all quadruple mutants with S108N, N51I, C59R, and I164L SNPs previously associated with pyrimethamine resistance but is now thought to convey CYC resistance (45). Further, among 17 case reports with known $P f d h f r$ status, failures within 3 days tended to occur among wild-type while failures at 7 days or later occurred among triple mutants (S108N, N51I, C59R), suggesting a possible role of this haplotype in ATQ/PG failures (41). None of the parasites in the present study had Pfcytb mutations at baseline, including the 4 recrudescences in the ATQ/PG arm. The declining efficacy from the present study (94\%) compared to the $2014-2015$ study (88\%) suggests increasing resistance from factors that need further exploration.

\section{Drug Pressure and Evolution}

The C580Y PfK13 mutation has been present in Western Cambodia since at least 2001 and a distinct C580Y haplotype, KEL 1, arose as early as 2007 in Western Cambodia, dominating that region by 2012 - 2013 (24). Pfplasmepsin 2/3 amplifications in Cambodia likely arose from the PLA1 haplotype as early as 2002 (48). In 2008, Western Cambodia saw both the adoption of DHA/PPQ as first-line therapy and the merger of KEL 1/PLA1 haplotypes associated with DHA/PPQ failures (48). This co-lineage has since spread outside Cambodia and by $2016-2017$ became dominant in several Southeast Asian nations (49). Interestingly, KEL 1 was associated with Pfmdr1 amplifications prior to 2008 , but by 2013 had mostly deamplified and rapidly gained Pfplasmepsin $2 / 3$ amplifications in Western Cambodia (48). Newer molecular markers for antimalarial failure are being identified, such as the new Pfcrt mutations (T93S, H97Y, F145I, I218F) (34, 49), the exonuclease E415G mutation (50, 51), and the quadruple Pfdhfr mutations (S108N, N51I, C59R, and I164L) (45). These mutations occurred on a genetic background of CVIET, KEL 1, and PLA1 and are fueled by drug pressure.

\section{Limitations}

The study was open label, but efforts were made to prevent randomization bias, and subjects received directly observed therapy. Twenty-one subjects (10\%) were withdrawn from final analysis of the primary endpoint due to loss of follow up (2), allergy (1), or $P$. vivax infection (18). Baseline levels of antimalarials were not drawn, which are indicators of private sector use of antimalarials, which may fuel the development of resistance. However, detectable PPQ levels at baseline are not always associated with clinical outcomes; recrudescence was associated in one study (27) but not another (25); baseline gametocytemia was associated in one study (52) but not another (34). Many biomarkers for antimalarial resistance were not yet validated or commonly available. PfK13 mutations were described in 2014 (24) and PC $_{1 / 2}$ estimation was described in 2011 (53). We did not test the isolates at the time for multiple markers of resistance to include PfCrt CVIET, though this is now recognized as the most common haplotype in the region $(7,38,40)$. The newer quadruple $P f C r t$ mutations were not described until 2019 (34). Pfplasmepsin 2/3 amplifications were associated with treatment failures of DHA/PPQ in 2017 (50, 54) and there is evidence that the E415G substitution of an exonuclease is a potential biomarker for PPQ resistance and failure $(50,51)$.

\section{Conclusion}

This 2009 - 2011 study was the first in Oddar Meanchey conducted during the transition to DHA/PPQ as first line therapy. This preceded a 2010 - 2011 study which saw $25 \%$ failure rate (38) and a 2012 - 2014 study which saw $50 \%$ failure rate $(8,25)$. In the interim, a decision was first made to adopt DHA/PPQ based on results from this study and others. However, in a short period of time, parasites rapidly acquired genetic mutations which conveyed PPQ resistance. 
The present declining efficacy of DHA/PPQ mirrors the declining copy numbers of Pfmdr1. Continued efforts to maximize non-pharmacologic therapies, use directly observed and publicly provided antimalarial therapy, surveillance studies to include molecular markers, and eradication of $P$. falciparum are needed in the region.

\section{Abbreviations}

ACPR Adequate Clinical and Parasitological Response

ACT Artemisinin Combination Therapy

AS/MQ Artesunate-Mefloquine

AS Artesunate

ATQ Atovaquone

ATQ/PG Atovaquone-Proguanil

CNM Cambodian National Center for Parasitology, Entomology, and Malaria Control

CQ Chloroquine

CRT Chloroquine Resistance Transporter

CYC Cycloguanil

DHA Dihydroartemisinin

DHA/PPQ Dihydroartemisinin-Piperaquine

$\mathrm{IC}_{50}$ Half-Maximal Inhibitory Concentration

K13 PfKelch 13

MDR1 Multidrug Resistance Protein 1

MQ Mefloquine

NAMRU-2 U.S. Naval Medical Research Unit 2

$\mathrm{PC}_{1 / 2}$ Parasite Clearance Half-Life

PG Proguanil

PPQ Piperaquine

SNP Single Nucleotide Polymorphism

TRAC Tracking Resistance to Artemisinin Collaboration

QN Quinine

WWARN Worldwide Antimalarial Resistance Network

WHO World Health Organization

\section{Declarations}

Ethics approval and consent to participate: The study protocol was approved by the Institutional Review Board of NAMRU-2 Jakarta (IRB\# 0702140230) and by the Cambodian National Ethical Committee for Health Research (NEHCR: 052 NEHCR). Consent was obtained from the subjects prior to enrolling into the trial.

Consent for publication: Not applicable

Availability of data and materials: The datasets used during this study are available from the corresponding author on reasonable request. The data may be shared with the Worldwide Antimalarial Research Network (WWARN). The protocol is available as an Additional File.

Competing interests: The authors have no competing interests to declare. 
Funding: Funding was provided by NAMRU-2 Jakarta work unit GEIS (D0016)/BEP (D0501), the Cambodian Ministry of Health, CNM, National Institute of Public Health (Cambodia), and Institute Pasteur de Cambodge.

Authors' contribution: DL, AR, DM, and WR designed the protocol and executed the study. DM, AR, DH, DL, SC, GC, and DS analyzed and interpreted the data. All authors contributed to writing the manuscript, read, and approved the final manuscript.

Acknowledgements: The authors would like to thank the staff of CNM, the Cambodian Ministry of Health, NAMRU-2, the Trapeang Prasat Health Center, and most importantly, the volunteers who participated and made this study possible.

Disclaimer: The opinions or assertions contained herein are the private opinions of the authors and are not to be construed as official or reflecting the views of the Department of Defense or the Uniformed Services University of the Health Sciences.

\section{References}

1. Trape JF. The public health impact of chloroquine resistance in Africa. Am J Trop Med Hyg. 2001;64(1-2 Suppl):12-7.

2. Zucker JR, Ruebush TK, Obonyo C, Otieno J, Campbell CC. The mortality consequences of the continued use of chloroquine in Africa: experience in Siaya, western Kenya. Am J Trop Med Hyg. 2003;68(4):386-90.

3. Na-Bangchang K, Congpuong K. Current malaria status and distribution of drug resistance in East and Southeast Asia with special focus to Thailand. Tohoku J Exp Med. 2007;211(2):99-113.

4. Myint HY, Ashley EA, Day NP, Nosten F, White NJ. Efficacy and safety of dihydroartemisinin-piperaquine. Trans R Soc Trop Med Hyg. 2007;101(9):858-66.

5. Wongsrichanalai C, Meshnick SR. Declining artesunate-mefloquine efficacy against falciparum malaria on the Cambodia-Thailand border. Emerg Infect Dis. 2008;14(5):716-9.

6. Nosten F, Luxemburger C, ter Kuile FO, Woodrow C, Eh JP, Chongsuphajaisiddhi T, et al. Treatment of multidrug-resistant Plasmodium falciparum malaria with 3-day artesunate-mefloquine combination. J Infect Dis. 1994;170(4):971-7.

7. Chaorattanakawee S, Saunders DL, Sea D, Chanarat N, Yingyuen K, Sundrakes S, et al. Ex Vivo Drug Susceptibility Testing and Molecular Profiling of Clinical Plasmodium falciparum Isolates from Cambodia from 2008 to 2013 Suggest Emerging Piperaquine Resistance. Antimicrob Agents Chemother. 2015;59(8):4631-43.

8. Saunders DL, Vanachayangkul P, Lon C, Program USAMMR, National Center for Parasitology E, Malaria C, et al. Dihydroartemisinin-piperaquine failure in Cambodia. N Engl J Med. 2014;371(5):484-5.

9. Leang R, Taylor WR, Bouth DM, Song L, Tarning J, Char MC, et al. Evidence of Plasmodium falciparum Malaria Multidrug Resistance to Artemisinin and Piperaquine in Western Cambodia: Dihydroartemisinin-Piperaquine Open-Label Multicenter Clinical Assessment. Antimicrob Agents Chemother. 2015;59(8):4719-26.

10. Vijaykadga S, Rojanawatsirivej C, Cholpol S, Phoungmanee D, Nakavej A, Wongsrichanalai C. In vivo sensitivity monitoring of mefloquine monotherapy and artesunate-mefloquine combinations for the treatment of uncomplicated falciparum malaria in Thailand in 2003. Trop Med Int Health. 2006;11(2):211-9.

11. Denis MB, Tsuyuoka R, Poravuth Y, Narann TS, Seila S, Lim C, et al. Surveillance of the efficacy of artesunate and mefloquine combination for the treatment of uncomplicated falciparum malaria in Cambodia. Trop Med Int Health. 2006;11(9):1360-6.

12. Rogers WO, Sem R, Tero T, Chim P, Lim P, Muth S, et al. Failure of artesunate-mefloquine combination therapy for uncomplicated Plasmodium falciparum malaria in southern Cambodia. Malar J. 2009;8:10.

13. Assessment. and Monitoring of Antimalarial Drug Efficacy for the Treatment of Uncomplicated Falciparum Malaria. Geneva: World Health Organization; 2003.

14. Wilson CM, Volkman SK, Thaithong S, Martin RK, Kyle DE, Milhous WK, et al. Amplification of pfmdr 1 associated with mefloquine and halofantrine resistance in Plasmodium falciparum from Thailand. Mol Biochem Parasitol. 1993;57(1):151-60.

15. Price RN, Dorsey G, Ashley EA, Barnes KI, Baird JK, d'Alessandro U, et al. World Antimalarial Resistance Network I: clinical efficacy of antimalarial drugs. Malar J. 2007;6:119.

16. Wilson PE, Alker AP, Meshnick SR. Real-time PCR methods for monitoring antimalarial drug resistance. Trends Parasitol. 2005;21(6):278-83.

17. Pickard AL, Wongsrichanalai C, Purfield A, Kamwendo D, Emery K, Zalewski C, et al. Resistance to antimalarials in Southeast Asia and genetic polymorphisms in pfmdr1. Antimicrob Agents Chemother. 2003;47(8):2418-23.

18. R. A language and environment for statistical computing. Vienna: R Foundation for Statistical Computing; 2018.

19. RStudio. Integrated Development for R. Boston: RStudio, PBC; 2020.

20. Update on artemisinin resistance - September. 2011: World Health Organization; 2011 [Available from:

https://www.who.int/.../resistance/updateonartemsininresistancesept2011.pdf.

21. Group WGS. Gametocyte carriage in uncomplicated Plasmodium falciparum malaria following treatment with artemisinin combination therapy: a systematic review and meta-analysis of individual patient data. BMC Med. 2016;14:79.

22. Fairhurst RM, Dondorp AM. Artemisinin-Resistant Plasmodium falciparum Malaria. Microbiol Spectr. 2016;4(3).

23. Amaratunga C, Sreng S, Suon S, Phelps ES, Stepniewska K, Lim P, et al. Artemisinin-resistant Plasmodium falciparum in Pursat province, western Cambodia: a parasite clearance rate study. Lancet Infect Dis. 2012;12(11):851-8.

Page $14 / 18$ 
24. Ariey F, Witkowski B, Amaratunga C, Beghain J, Langlois AC, Khim N, et al. A molecular marker of artemisinin-resistant Plasmodium falciparum malaria. Nature. 2014;505(7481):50-5.

25. Spring MD, Lin JT, Manning JE, Vanachayangkul P, Somethy S, Bun R, et al. Dihydroartemisinin-piperaquine failure associated with a triple mutant including kelch13 C580Y in Cambodia: an observational cohort study. Lancet Infect Dis. 2015;15(6):683-91.

26. Ashley EA, Dhorda M, Fairhurst RM, Amaratunga C, Lim P, Suon S, et al. Spread of artemisinin resistance in Plasmodium falciparum malaria. N Engl J Med. 2014;371(5):411-23.

27. Amaratunga C, Lim P, Suon S, Sreng S, Mao S, Sopha C, et al. Dihydroartemisinin-piperaquine resistance in Plasmodium falciparum malaria in Cambodia: a multisite prospective cohort study. Lancet Infect Dis. 2016;16(3):357-65.

28. Witkowski B, Amaratunga C, Khim N, Sreng S, Chim P, Kim S, et al. Novel phenotypic assays for the detection of artemisinin-resistant Plasmodium falciparum malaria in Cambodia: in-vitro and ex-vivo drug-response studies. Lancet Infect Dis. 2013;13(12):1043-9.

29. Boudreau EF, Webster HK, Pavanand K, Thosingha L. Type II mefloquine resistance in Thailand. Lancet. 1982;2(8311):1335.

30. Price RN, Uhlemann AC, Brockman A, McGready R, Ashley E, Phaipun L, et al. Mefloquine resistance in Plasmodium falciparum and increased pfmdr1 gene copy number. Lancet. 2004;364(9432):438-47.

31. Alker AP, Lim P, Sem R, Shah NK, Yi P, Bouth DM, et al. Pfmdr1 and in vivo resistance to artesunate-mefloquine in falciparum malaria on the CambodianThai border. Am J Trop Med Hyg. 2007;76(4):641-7.

32. Dondorp AM, Nosten F, Yi P, Das D, Phyo AP, Tarning J, et al. Artemisinin resistance in Plasmodium falciparum malaria. N Engl J Med. 2009;361(5):45567.

33. Leang R, Barrette A, Bouth DM, Menard D, Abdur R, Duong S, et al. Efficacy of dihydroartemisinin-piperaquine for treatment of uncomplicated Plasmodium falciparum and Plasmodium vivax in Cambodia, 2008 to 2010. Antimicrob Agents Chemother. 2013;57(2):818-26.

34. van der Pluijm RW, Imwong M, Chau NH, Hoa NT, Thuy-Nhien NT, Thanh NV, et al. Determinants of dihydroartemisinin-piperaquine treatment failure in Plasmodium falciparum malaria in Cambodia, Thailand, and Vietnam: a prospective clinical, pharmacological, and genetic study. Lancet Infect Dis. 2019;19(9):952-61.

35. van der Pluijm RW, Tripura R, Hoglund RM, Pyae Phyo A, Lek D, Ul Islam A, et al. Triple artemisinin-based combination therapies versus artemisinin-based combination therapies for uncomplicated Plasmodium falciparum malaria: a multicentre, open-label, randomised clinical trial. Lancet. 2020;395(10233):1345-60.

36. Wootton JC, Feng X, Ferdig MT, Cooper RA, Mu J, Baruch DI, et al. Genetic diversity and chloroquine selective sweeps in Plasmodium falciparum. Nature. 2002;418(6895):320-3.

37. Awasthi G, Prasad GB, Das A. Population genetic analyses of Plasmodium falciparum chloroquine receptor transporter gene haplotypes reveal the evolutionary history of chloroquine-resistant malaria in India. Int J Parasitol. 2011;41(7):705-9.

38. Lon C, Manning JE, Vanachayangkul P, So M, Sea D, Se Y, et al. Efficacy of two versus three-day regimens of dihydroartemisinin-piperaquine for uncomplicated malaria in military personnel in northern Cambodia: an open-label randomized trial. PLoS One. 2014;9(3):e93138.

39. Chaorattanakawee S, Lon C, Jongsakul K, Gawee J, Sok S, Sundrakes S, et al. Ex vivo piperaquine resistance developed rapidly in Plasmodium falciparum isolates in northern Cambodia compared to Thailand. Malar J. 2016;15(1):519.

40. Veiga MI, Dhingra SK, Henrich PP, Straimer J, Gnädig N, Uhlemann AC, et al. Globally prevalent PfMDR1 mutations modulate Plasmodium falciparum susceptibility to artemisinin-based combination therapies. Nat Commun. 2016;7:11553.

41. Staines HM, Burrow R, Teo BH, Chis Ster I, Kremsner PG, Krishna S. Clinical implications of Plasmodium resistance to atovaquone/proguanil: a systematic review and meta-analysis. J Antimicrob Chemother. 2018;73(3):581-95.

42. Wojnarski M, Lon C, Vanachayangkul P, Gosi P, Sok S, Rachmat A, et al. Atovaquone-Proguanil in Combination With Artesunate to Treat MultidrugResistant P. falciparum Malaria in Cambodia: An Open-Label Randomized Trial. Open Forum Infect Dis. 2019;6(9):ofz314.

43. Saunders DL, Chaorattanakawee S, Gosi P, Lanteri C, Somethy S, Kuntawunginn W, et al. Atovaquone-Proguanil Remains a Potential Stopgap Therapy for Multidrug-Resistant Plasmodium falciparum in Areas along the Thai-Cambodian Border. Antimicrob Agents Chemother. 2015;60(3):1896-8.

44. Maude RJ, Nguon C, Dondorp AM, White LJ, White NJ. The diminishing returns of atovaquone-proguanil for elimination of Plasmodium falciparum malaria: modelling mass drug administration and treatment. Malar J. 2014;13:380.

45. Lin JT, Waltmann A, Moser KA, Park Z, Na YB, Aydemir O, et al. Selection of Cytochrome. Antimicrob Agents Chemother. 2021;65(3).

46. Canfield CJ, Pudney M, Gutteridge WE. Interactions of atovaquone with other antimalarial drugs against Plasmodium falciparum in vitro. Exp Parasitol. 1995;80(3):373-81.

47. van Vugt M, Leonardi E, Phaipun L, Slight T, Thway KL, McGready R, et al. Treatment of uncomplicated multidrug-resistant falciparum malaria with artesunate-atovaquone-proguanil. Clin Infect Dis. 2002;35(12):1498-504.

48. Amato R, Pearson RD, Almagro-Garcia J, Amaratunga C, Lim P, Suon S, et al. Origins of the current outbreak of multidrug-resistant malaria in southeast Asia: a retrospective genetic study. Lancet Infect Dis. 2018;18(3):337-45.

49. Hamilton WL, Amato R, van der Pluijm RW, Jacob CG, Quang HH, Thuy-Nhien NT, et al. Evolution and expansion of multidrug-resistant malaria in southeast Asia: a genomic epidemiology study. Lancet Infect Dis. 2019;19(9):943-51.

50. Amato R, Lim P, Miotto O, Amaratunga C, Dek D, Pearson RD, et al. Genetic markers associated with dihydroartemisinin-piperaquine failure in Plasmodium falciparum malaria in Cambodia: a genotype-phenotype association study. Lancet Infect Dis. 2017;17(2):164-73.

51. Parobek CM, Parr JB, Brazeau NF, Lon C, Chaorattanakawee S, Gosi P, et al. Partner-Drug Resistance and Population Substructuring of ArtemisininResistant Plasmodium falciparum in Cambodia. Genome Biol Evol. 2017;9(6):1673-86.

Page $15 / 18$ 
52. Lin JT, Lon C, Spring MD, Sok S, Chann S, Ittiverakul M, et al. Single dose primaquine to reduce gametocyte carriage and Plasmodium falciparum transmission in Cambodia: An open-label randomized trial. PLoS One. 2017;12(6):e0168702.

53. Flegg JA, Guerin PJ, White NJ, Stepniewska K. Standardizing the measurement of parasite clearance in falciparum malaria: the parasite clearance estimator. Malar J. 2011;10:339.

54. Witkowski B, Duru V, Khim N, Ross LS, Saintpierre B, Beghain J, et al. A surrogate marker of piperaquine-resistant Plasmodium falciparum malaria: a phenotype-genotype association study. Lancet Infect Dis. 2017;17(2):174-83.

55. Noedl H, Se Y, Schaecher K, Smith BL, Socheat D, Fukuda MM, et al. Evidence of artemisinin-resistant malaria in western Cambodia. N Engl J Med. 2008;359(24):2619-20.

56. Noedl H, Se Y, Sriwichai S, Schaecher K, Teja-Isavadharm P, Smith B, et al. Artemisinin resistance in Cambodia: a clinical trial designed to address an emerging problem in Southeast Asia. Clin Infect Dis. 2010;51(11):e82-9.

57. Rueangweerayut R, Phyo AP, Uthaisin C, Poravuth Y, Binh TQ, Tinto H, et al. Pyronaridine-artesunate versus mefloquine plus artesunate for malaria. N Engl J Med. 2012;366(14):1298-309.

58. Bethell D, Se Y, Lon C, Tyner S, Saunders D, Sriwichai S, et al. Artesunate dose escalation for the treatment of uncomplicated malaria in a region of reported artemisinin resistance: a randomized clinical trial. PLoS One. 2011;6(5):e19283.

59. Leang R, Canavati SE, Khim N, Vestergaard LS, Borghini Fuhrer I, Kim S, et al. Efficacy and Safety of Pyronaridine-Artesunate for Treatment of Uncomplicated Plasmodium falciparum Malaria in Western Cambodia. Antimicrob Agents Chemother. 2016;60(7):3884-90.

\section{Figures}

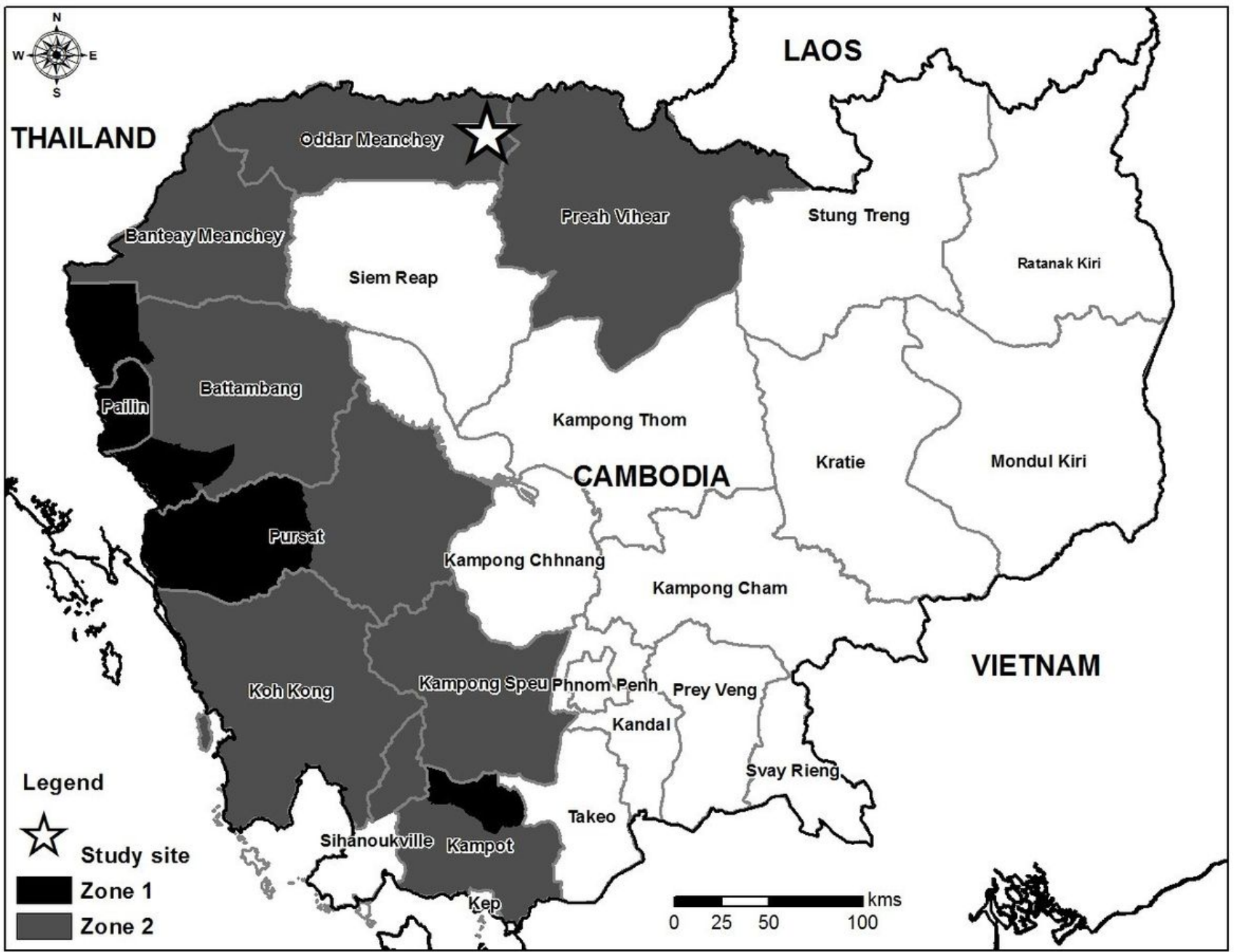

Figure 1

Map of Cambodia with Phase 1 and 2 Zones

Site of the 2009 study in relation to previously established malaria containment Zones 1 and 2 . Zone 1 was considered the highest risk and had already switched to the use of DHA/PPQ. In addition, there were monitored mass screening and treatment activities using ATQ/PG for PCR-identified subclinical P.falciparum malaria cases. 


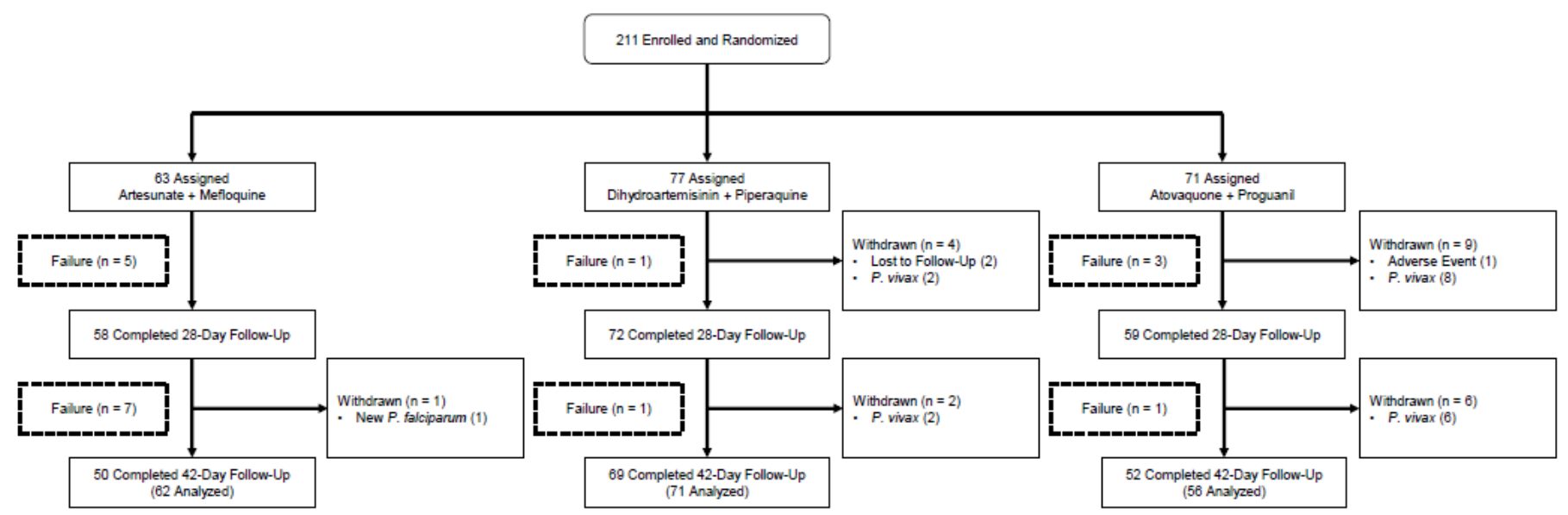

Figure 2

Consort Flow Diagram

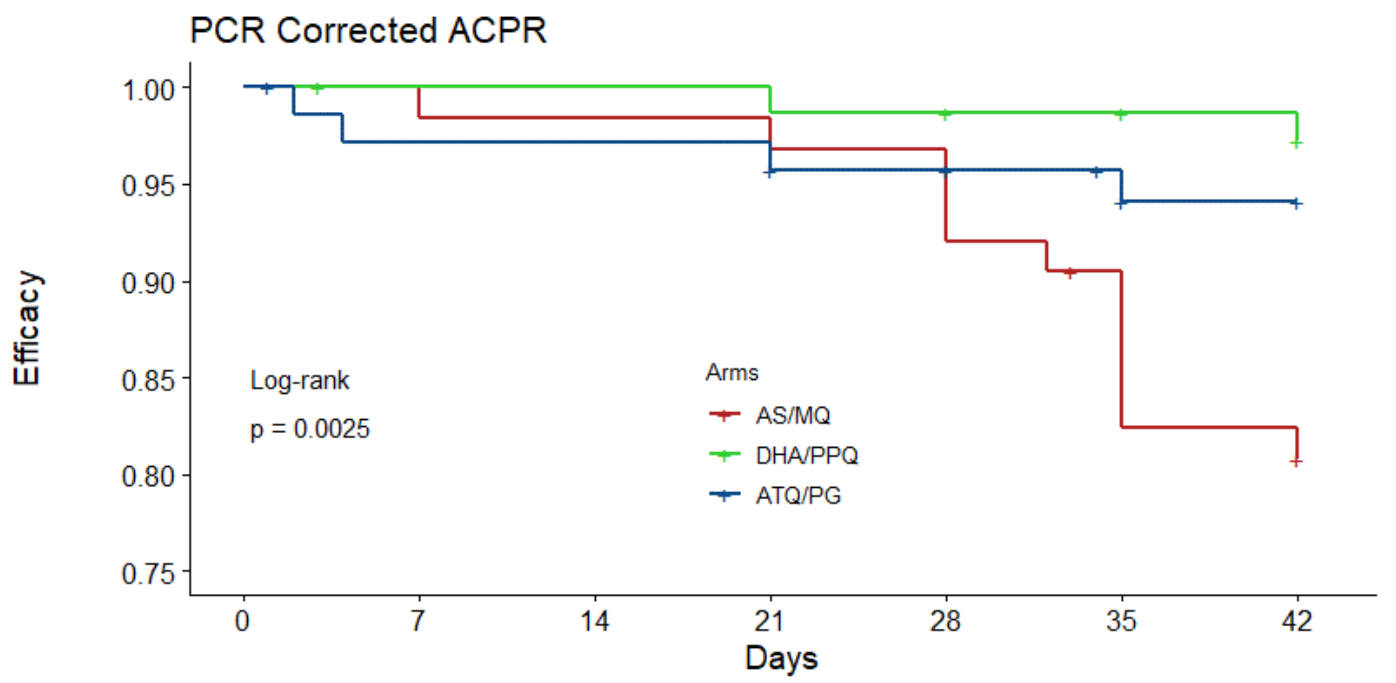

Number at risk

\begin{tabular}{|c|c|c|c|c|c|c|c|}
\hline 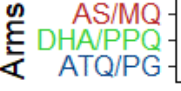 & $\begin{array}{l}63 \\
77 \\
71 \\
\end{array}$ & $\begin{array}{l}63 \\
75 \\
68 \\
\end{array}$ & $\begin{array}{l}62 \\
75 \\
68 \\
\end{array}$ & $\begin{array}{l}62 \\
75 \\
68 \\
\end{array}$ & $\begin{array}{l}61 \\
74 \\
65 \\
\end{array}$ & $\begin{array}{l}56 \\
72 \\
58 \\
\end{array}$ & $\begin{array}{l}51 \\
70 \\
52 \\
\end{array}$ \\
\hline & 0 & 7 & 14 & $\begin{array}{l}21 \\
\text { Days }\end{array}$ & 28 & 35 & 42 \\
\hline
\end{tabular}

Figure 3

\section{Kaplan Meier Survival Estimates}

Kaplan Meier survival analysis was performed for each of the three study groups to compare parasite-free survival between AS/MQ (red), DHA/PPQ (green) and ATQ/PG (blue). Tic marks on each curve indicate a censored subject. 


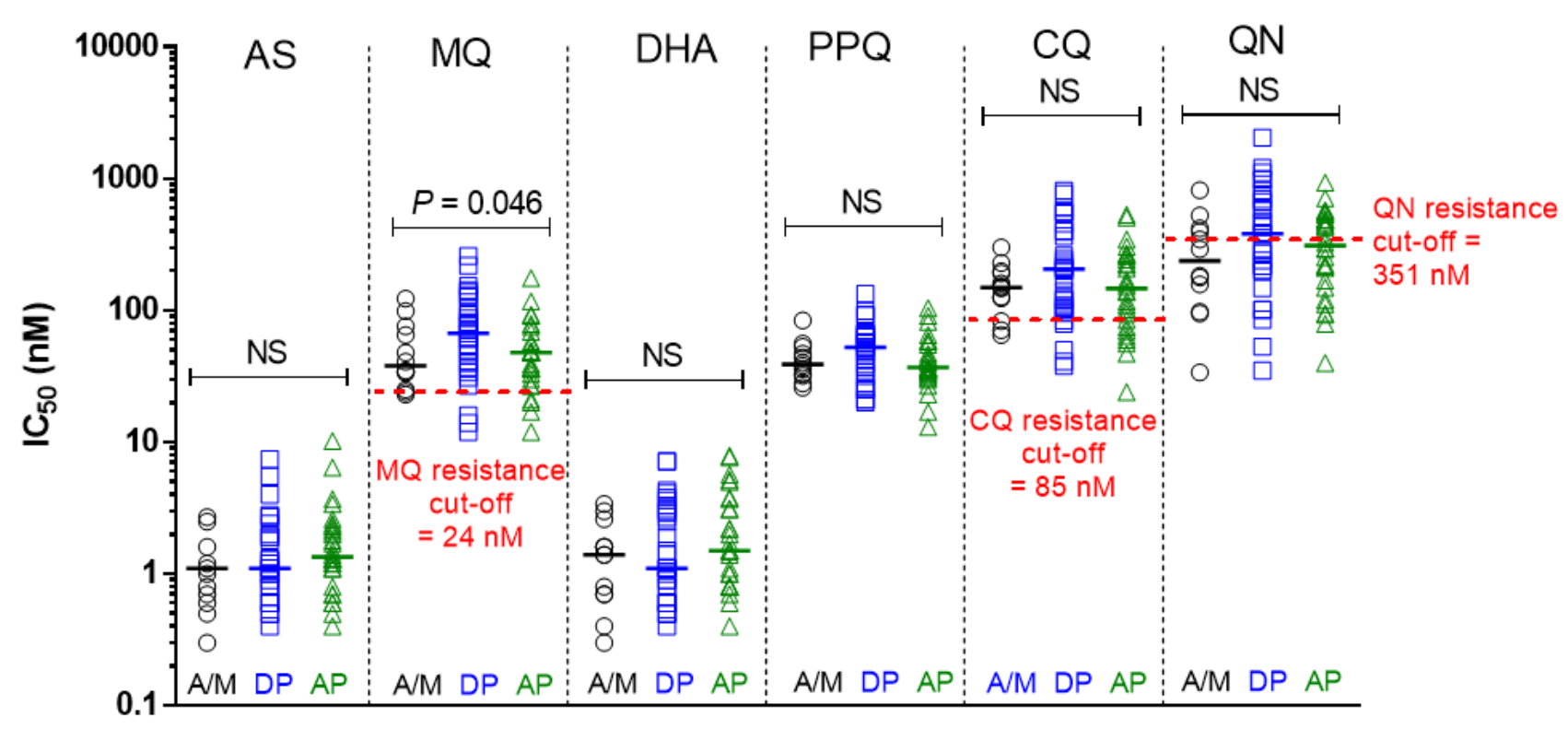

Figure 4

\section{Comparing in vitro Parasite Drug Resistance against Common Antimalarials}

Samples from 83 subject isolates with $>6,400$ parasites $/ \mu \mathrm{L}$ were run in a classical isotopic $P$. falciparum in vitro drug resistance assay. In vitro resistance was calculated as the $50 \%$ inhibitory concentrations $\left(\mathrm{IC}_{50}\right)$ based on serial dilutions of artesunate $(\mathrm{AS})$, mefloquine (MQ), dihydroartemisin (DHA), piperaquine (PPQ), chloroquine (CQ) and quinine (QN). Resistance cut-offs established for the assays where known were used (MQ, CQ and QN). Resistance was compared between the three treatment groups (artesunate-mefloquine (A/M) in black circles; dihydroartemisinin-piperaquine (DP) in blue squares, and atovaquone-proguanil (AP) in green triangles.

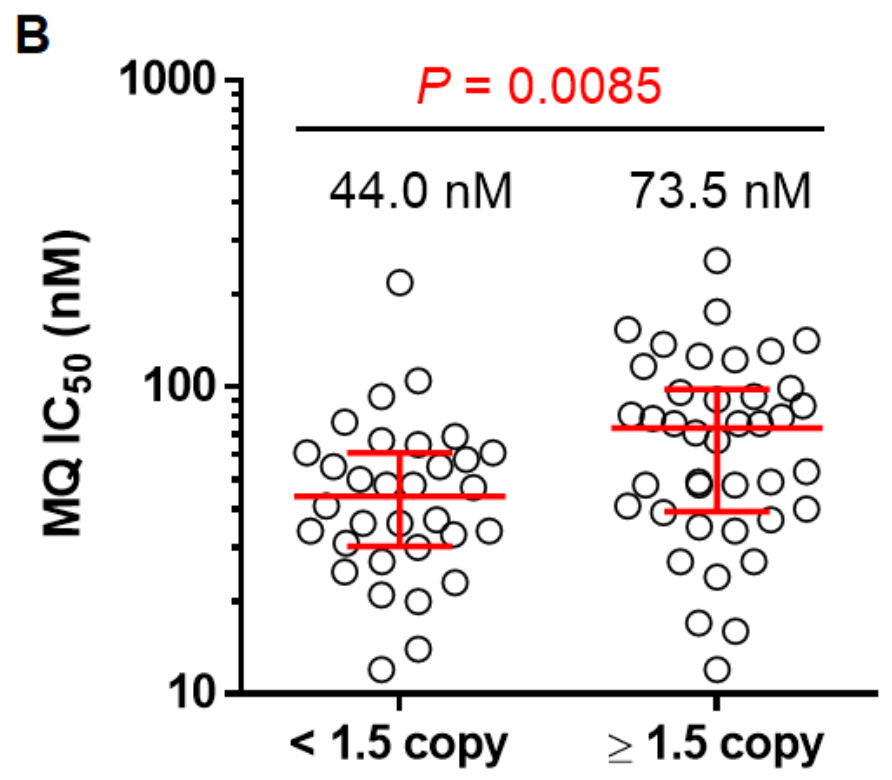

Figure 5

Relationship between Mefloquine $\mathrm{IC}_{50}$ and $P f m d r 1$ Status

A. Correlation between MQ IC ${ }_{50}$ and $P f m d r 1$ copy number. B. Mefloquine $I_{50}$ was elevated in parasites with $\geq 1.5$ copies of $P f m d r 1$. 